\title{
The Aubry set for a version of the Vlasov equation
}

\author{
Ugo Bessi
}

\begin{abstract}
We check that several properties of the Aubry set, first proven for finite-dimensional Lagrangians by Mather and Fathi, continue to hold in the case of the infinitely many interacting particles of the Vlasov equation on the circle.
\end{abstract}

Mathematics Subject Classification. Primary 37J50; Secondary 35Q83.

Keywords. Vlasov equation, Aubry-Mather theory.

\section{Introduction}

The Vlasov equation on the circle governs the motion of a group of particles on $S^{1}:=\frac{\mathbf{R}}{\mathbf{Z}}$ under the action of an external potential $V(t, x)$ and a mutual interaction $W$. More precisely, we let $I=[0,1)$, we lift our particles to $\mathbf{R}$, and we parametrize them at time $t$ by a function $\sigma_{t} \in L^{2}(I, \mathbf{R})$; we require that $\sigma_{t}$ satisfies the differential equation in $L^{2}(I, \mathbf{R})$

$$
\ddot{\sigma}_{t} z=-V^{\prime}\left(t, \sigma_{t} z\right)-\int_{I} W^{\prime}\left(\sigma_{t} z-\sigma_{t} \bar{z}\right) \mathrm{d} \bar{z} . \quad(O D E)_{L a g}
$$

Our standing hypotheses on the potentials $V$ and $W$ are

- $V \in C^{2}\left(S^{1} \times S^{1}\right), W \in C^{2}\left(S^{1}\right)$; moreover $W$, seen as a function on $\mathbf{R}$, is even; up to adding a constant, we can suppose that $W(0)=0$.

There is an element of arbitrariness in choosing the lift of the particles to $\mathbf{R}$ and in parametrizing them; that's why we are less interested in the evolution of the labelling $\sigma_{t}$ than in the evolution of the measure it induces. In other words, we want to study the measures on $S^{1} \times \mathbf{R}$ given by $\mu_{t}:=\left(\pi \circ \sigma_{t}, \dot{\sigma}_{t}\right)_{\sharp} \nu_{0}$, where $\nu_{0}$ denotes the Lebesgue measure on $I, \pi: \mathbf{R} \rightarrow S^{1}$ is the natural projection and $(\cdot)_{\sharp}$ denotes the push-forward. A standard calculation shows that, if $\sigma_{t}$ satisfies $(O D E)_{L a g}$, then $\mu_{t}$ satisfies, in the weak sense,

$$
\partial_{t} \mu_{t}+v \partial_{x} \mu_{t}=\partial_{v}\left(\mu_{t} \partial_{x} P_{t}\right) \quad(O D E)_{\text {meas }}
$$

This work was partially supported by the PRIN2009 grant "Critical Point Theory and Perturbative Methods for Nonlinear Differential Equations. 
where

$$
P_{t}(x)=V(t, x)+\int_{S^{1} \times \mathbf{R}} W(x-\bar{x}) \mathrm{d} \mu_{t}(\bar{x}, v)=V(t, x)+\int_{I} W\left(x-\sigma_{t} \bar{z}\right) \mathrm{d} \bar{z} .
$$

Problem $(O D E)_{\text {meas }}$ (see $\left.[9,10]\right)$ is Lagrangian; actually, many results of Aubry-Mather theory can be extended to curves of measures which are "minimal" in a suitable way. Here, however, we follow the approach of [8]: we are going to work with $(O D E)_{L a g}$, keeping track of its symmetries. Quotienting $(O D E)_{\text {Lag }}$ by its symmetry group, we shall get a problem equivalent to $(O D E)_{\text {meas }}$. Though in this paper we restrict ourselves to the one-dimensional situation, we recall that Nurbekian [14] has extended the results of [8] about minimal parametrizations to tori of any dimension.

The aim of this paper is to do a few simple checks, showing that many features of Aubry-Mather theory persist in this setting; actually, we shall check that the main theorems of [7] continue to hold. In Sect. 1, we recall the main results of [8] on $(O D E)_{\text {Lag }}$ and its symmetries; in Sect. 2, following $[7,8,10]$, we define the Hopf-Lax semigroup and we show that it has fixed points. We also show that the value function satisfies the Hamilton-Jacobi equation on $L^{2}$. In Sect. 3, we show that $(O D E)_{L a g}$ admits invariant measures minimal in the sense of Mather; as a consequence, we can define Mather's conjugate actions $\alpha$ and $\beta$. In Sect. 4, we recall two different definitions of the Aubry set, one of Mather's and the other of Fathi's; we show that, also in this case, the two definitions coincide. In Sect. 5, we shall show that $(O D E)_{\text {meas }}$ admits a solution $\mu_{t}$ which is periodic (i.e. $\mu_{0}=\mu_{1}$ ) and has irrational rotation number. We shall see that, as a consequence of the KAM theorem, if the rotation number $\omega$ is sufficiently irrational, and $V$ and $W$ are sufficiently regular and small (depending on $\omega$ ), then $\mu_{t}$ has a smooth density.

\section{Notation and preliminaries}

Since $V$ and $W$ are periodic, we have that $(O D E)_{L a g}$ is invariant by the action of $L_{\mathbf{Z}}^{2}:=L^{2}(I, \mathbf{Z})$; in other words, if $\sigma_{t}$ is a solution and $h \in L_{\mathbf{Z}}^{2}$, then $\sigma_{t}+h$ is a solution too. Moreover, $(O D E)_{L a g}$ is invariant by the group $G$ of the measure-preserving transformations of $I$ into itself; indeed, such maps do not change the value of the integral defining $P_{t}(x)$. An idea of [8] is to quotient $L^{2}(I)$ by these two groups; we recall from [8] some facts about this quotient.

We shall denote by $\|\cdot\|$ the norm on $L^{2}(I),\langle\cdot, \cdot\rangle$ the internal product. We set

$$
\mathbf{T}:=\frac{L^{2}(I)}{L_{\mathbf{Z}}^{2}(I)} .
$$

The space $\mathbf{T}$ is metric, with distance between the equivalence classes $[M]$ and $[\bar{M}]$ given by

$$
\operatorname{dist}_{\mathbf{Z}}([M],[\bar{M}])=\inf _{Z \in L_{\mathbf{Z}}^{2}}\|M-\bar{M}-Z\|=\left|\left\|M-\left.\bar{M}\right|_{S^{1}}\right\|\right.
$$

where

$$
|m|_{S^{1}}:=\min _{k \in \mathbf{Z}}|m+k| .
$$


We note that, for each $x \in I$, we can measurably choose $Z x \in \mathbf{Z}$ such that $|M x-\bar{M} x-Z x|=|M x-\bar{M} x|_{S^{1}}$; as a consequence, we get that the inf in the definition of dist $_{\mathbf{Z}}$ is a minimum; we also get the second equality above.

Let Group denote the group of the measure-preserving transformations of $I$ with measurable inverse; for $M, \bar{M} \in L^{2}(I)$ we set

$$
\operatorname{dist}_{\text {weak }}(M, \bar{M})=\inf _{G \in \operatorname{Group}} \operatorname{dist}_{\mathbf{Z}}(M \circ G, \bar{M}) .
$$

This yields that $M$ and $M \circ G$, which we would like to consider equivalent, have zero distance; however, if we say that $M \simeq \bar{M}$ when $\bar{M}=M \circ G$ for some $G \in$ Group, then the equivalence classes are not closed in $\mathbf{T}$, essentially because the inf in the definition of dist weak is not a minimum: it is possible (see [8]) that $\operatorname{dist}(M, \bar{M})=0$ even if $M$ and $\bar{M}$ are not equivalent. But we can consider their closure if we look at the equivalence relation from the right point of view, i.e. that of the measure induced by $M$.

We denote by Meas the space of Borel probability measures on $S^{1}$, and we let $\pi: \mathbf{R} \rightarrow S^{1}$ be the natural projection. We introduce the map

$$
\Phi: L^{2}(I) \rightarrow \text { Meas, } \quad \Phi: M \rightarrow(\pi \circ M)_{\sharp} \nu_{0}
$$

where $(\cdot)_{\sharp}$ denotes push-forward and $\nu_{0}$ is the Lebesgue measure on $I$. We note that $\Phi$ is invariant under the action of $L_{\mathbf{Z}}^{2}$ and Group; in other words, if $Z \in L_{\mathbf{Z}}^{2}$ and $G \in$ Group, then $\Phi(u)=\Phi((u+Z) \circ G)$. We say that $M \simeq \bar{M}$ if $\Phi(M)=\Phi(\bar{M})$. We set $\mathbf{S}:=\frac{L^{2}(I)}{\simeq}$; on this space, we consider the metric

$$
\operatorname{dist}_{\mathbf{S}}([M],[\bar{M}])=\inf \left\{\left\|M^{*}-\bar{M}^{*}\right\|: M^{*} \in[M], \quad \bar{M}^{*} \in[\bar{M}]\right\} .
$$

The infimum above is a minimum: one can always find a minimal couple $\left(M^{*}, \bar{M}^{*}\right)$ with $M$ monotone and taking values in [0,1], and $\bar{M}$ monotone and taking values in $\left[-\frac{3}{2}, \frac{3}{2}\right]$. Now $\mathbf{S}$ is isometric to the space of Borel probability measures on $S^{1}$ with the 2-Wasserstein distance; in particular, it is a compact space.

Another fact proven in $[8]$ is that $\operatorname{dist}_{\text {weak }}(M, \bar{M})=\operatorname{dist}_{\mathbf{S}}([M],[\bar{M}])$.

By Proposition 2.9 of [8], which we copy below, the $L_{\mathbf{Z}}^{2}$-equivariant (or $L_{\mathbf{Z}}^{2}$-equivariant and Group-equivariant) closed forms on $L^{2}(I)$ have a particularly simple structure: the first equivariant cohomology group of $L^{2}(I)$ is $\mathbf{R}$.

Proposition 1.1. Let $S: L^{2}(I) \rightarrow \mathbf{R}$ be $C^{1}$.

1. If $\mathrm{d} S$ is $L_{\mathbf{Z}}^{2}$-periodic in the sense that $\mathrm{d}_{M+Z} S=\mathrm{d}_{M} S$ for all $Z \in L_{\mathbf{Z}}^{2}(I)$, then there is a unique $C \in L^{2}(I)$ and a function $s: L^{2}(I) \rightarrow \mathbf{R}$, of class $C^{1}$ and $L_{\mathbf{Z}}^{2}$-periodic, such that

$$
S(M)=s(M)+\langle C, M\rangle .
$$

2. If, in addition, : $M \rightarrow \mathrm{d}_{M} S$ is rearrangement-invariant (i.e. if $\mathrm{d}_{M} S=$ $\mathrm{d}_{M \circ G} S$ for all $G \in$ Group), then $C$ is constant and $s$ is rearrangementinvariant. 
In view of the lemma above, for $c \in \mathbf{R}$ we define the Lagrangian $\mathcal{L}_{c}$ as

$$
\begin{aligned}
\mathcal{L}_{c}: S^{1} \times L^{2}(I) \times L^{2}(I) \rightarrow \mathbf{R}, \quad \mathcal{L}_{c}(t, M, N)= & \frac{1}{2}\|N\|^{2}-\langle c, N\rangle-\mathcal{V}(t, M) \\
& -\mathcal{W}(M)
\end{aligned}
$$

where

$$
\mathcal{V}(t, M)=\int_{I} V(t, M x) \mathrm{d} x, \quad \text { and } \quad \mathcal{W}(M)=\frac{1}{2} \int_{I \times I} W\left(M x-M x^{\prime}\right) \mathrm{d} x \mathrm{~d} x^{\prime} .
$$

In order to define the $c$-minimal orbits of $\mathcal{L}$, we let $K \subset \mathbf{R}$ be an interval; following [1], we say that $u \in L^{1}\left(K, L^{2}(I)\right)$ is absolutely continuous if there is $\dot{u} \in L^{1}\left(K, L^{2}(I)\right)$ such that, for any $\phi \in C_{0}^{1}(K, \mathbf{R})$, we have that

$$
\int_{K} u_{t}(x) \dot{\phi}(t) \mathrm{d} t=-\int_{K} \dot{u}_{t}(x) \phi(t) \mathrm{d} t
$$

The equality above is an equality in $L^{2}(I)$, i.e. it holds for a.e. $x \in I$; however, it is easy to see that the exceptional set does not depend on $\phi$, and thus that, for a.e. $x$, the map $: t \rightarrow u_{t}(x)$ is A. C. with derivative $\dot{u}_{t}(x)$. We shall denote by $A C\left(K, L^{2}(I)\right)$ the class of A. C. functions from $K$ to $L^{2}(I)$.

Let $c \in \mathbf{R}$; we say that $\sigma \in A C\left(K, L^{2}(I)\right)$ is $c$-minimal for $\mathcal{L}$ if, for any interval $\left[t_{0}, t_{1}\right] \subset K$ and any $\tilde{\sigma} \in A C\left(\left(t_{0}, t_{1}\right), L^{2}(I)\right)$ satisfying

$$
\tilde{\sigma}_{t_{1}}-\sigma_{t_{1}} \in L_{\mathbf{Z}}^{2}(I) \text { and } \tilde{\sigma}_{t_{2}}-\sigma_{t_{2}} \in L_{\mathbf{Z}}^{2}(I),
$$

we have that

$$
\int_{t_{0}}^{t_{1}} \mathcal{L}_{c}\left(t, \sigma_{t}, \dot{\sigma}_{t}\right) \mathrm{d} t \leq \int_{t_{0}}^{t_{1}} \mathcal{L}_{c}\left(t, \tilde{\sigma}_{t}, \dot{\tilde{\sigma}}_{t}\right) \mathrm{d} t
$$

We forego the standard proof that $c$-minimal orbits solve $(O D E)_{L a g}$.

Let now $n \in \mathbf{N}$, and let $\mathcal{A}_{n}$ be the $\sigma$-algebra on $I$ generated by the intervals $\left[\frac{i}{n}, \frac{i+1}{n}\right)$ with $i \in(0, \ldots, n-1)$; we call $\mathcal{C}_{n}$ the closed subspace of the $\mathcal{A}_{n}$-measurable functions of $L^{2}(I)$, and we denote by $P_{n}: L^{2}(I) \rightarrow \mathcal{C}_{n}$ the orthogonal projection. We have a bijection

$$
D_{n}: \mathbf{R}^{n} \rightarrow \mathcal{C}_{n}, \quad D_{n}:\left(q_{1}, \ldots, q_{n}\right) \rightarrow \sum_{i=0}^{n-1} q_{i} 1_{\left[\frac{i}{n}, \frac{i+1}{n}\right)}(x) .
$$

We also note that the space $S^{1} \times \mathcal{C}_{n} \times \mathcal{C}_{n}$ is invariant by the EulerLagrange flow of $(O D E)_{\text {Lag }}$.

\section{The Hopf-Lax semigroup}

Definition. Let us denote by $C_{\text {Group }}(\mathbf{T})$ the set of functions $U \in C\left(L^{2}(I), \mathbf{R}\right)$ which are $L_{\mathbf{Z}}^{2}$ and Group equivariant. It is standard (Proposition 2.8 of [8]) each $U \in C_{\text {Group }}(\mathbf{T})$ quotients to a continuous function on the compact space $\mathbf{S}$; in particular, it is bounded.

Given $M \in L^{2}(I), U \in C_{\text {Group }}(\mathbf{T})$ and $t>0$, we define 


$$
\begin{gathered}
\left(A_{c}^{t} U\right)(M)=\inf \left\{\int_{0}^{t} \mathcal{L}_{c}\left(s, \sigma_{s}, \dot{\sigma}_{s}\right) \mathrm{d} s+U\left(\sigma_{0}\right): \sigma \in A C\left([0, t], L^{2}(I)\right),\right. \\
\left.\sigma_{t}=M\right\} .
\end{gathered}
$$

We shall denote by Mon the space of the maps $\sigma: I \rightarrow \mathbf{R}$ which are monotone increasing and satisfy $\sigma(1-) \leq \sigma(0)+1$. We endow Mon with the topology it inherits from $L^{2}(I)$, which turns it into a locally compact space.

We group together the statements of a few lemmas of [8] and [10]; for a slightly different proof, point 1 is lemma 2.1 of [5], point 2 is lemma 2.8, point 4 Proposition 2.2.

Proposition 2.1. Let $U \in C_{\mathrm{Group}}(\mathbf{T})$, let $t>0$ and let $A_{c}^{t} U: L^{2}(I) \rightarrow \mathbf{R}$ be defined by (2.1); then, the following statements hold.

1. $A_{c}^{t} U$ is $L_{\mathbf{Z}}^{2}$ and Group-equivariant.

2. $A_{c}^{t} U$ is $L(t)$-Lipschitz for dist weak (or for dist ${ }_{\mathbf{S}}$, since we have seen that the two distances coincide). The constant $L(t)$ does not depend on $U$. Moreover, $L(t) \leq L$ for $t \geq 1$.

3. As a consequence of 1 and $2, A_{c}^{t} U \in C_{\text {Group }}(\mathbf{T})$.

4. Let $M \in M$ on; then, the inf in (2.1) is a minimum; more precisely, there is $\sigma \in A C\left([0, t], L^{2}(I)\right)$ with $\sigma_{t}=M, \sigma_{s} \in$ Mon for $s \in[0, t]$ and such that

$$
\left(A_{c}^{t} U\right)(M)=\int_{0}^{t} \mathcal{L}_{c}\left(s, \sigma_{s}, \dot{\sigma}_{s}\right) \mathrm{d} s+U\left(\sigma_{0}\right) .
$$

The function $\sigma$ is c-minimal on $(0, t)$ and solves $(O D E)_{\text {Lag }}$.

5. Since $\mathcal{L}_{c}$ is one-periodic in time, $A_{c}^{t}$ has the semigroup property on the integers: in other words, if $t>0$ and $s \in \mathbf{N}$, then

$$
A_{c}^{t+s} U=A_{c}^{t}\left(A_{c}^{s} U\right) \text {. }
$$

Let $l \in \mathbf{R}$; by point 3 of the last lemma, we can define a map

$$
\begin{gathered}
\Lambda_{c, \lambda}: C_{\text {Group }}(\mathbf{T}) \rightarrow C_{\text {Group }}(\mathbf{T}) \\
\Lambda_{c, \lambda}: U \rightarrow\left(A_{c}^{1} U\right)(\cdot)+\lambda .
\end{gathered}
$$

It follows immediately from the definition of $A_{c}^{1} U$ that

- $\Lambda_{c, \lambda}$ is monotone, i.e., if $U_{1} \leq U_{2}$, then $\Lambda_{c, \lambda} U_{1} \leq \Lambda_{c, \lambda} U_{1}$.

- If $a \in \mathbf{R}$, then $\Lambda_{c, \lambda}(U+a)=\Lambda_{c, \lambda} U+a$. These two facts easily imply that

- $\Lambda_{c, \lambda}$ is continuous (actually, 1-Lipschitz) from $C_{\text {Group }}(\mathbf{T})$ to itself, if we put on $C_{\text {Group }}(\mathbf{T})$ the sup norm.

Again, we refer the reader to [8,10] (or to [7], since the finite dimensional proof is the same) for the next lemma; in [5], point 1) is Proposition 2.11. Point 2 follows in a standard way by point 1 and the semigroup property.

Proposition 2.2. $\quad$ 1. There is a unique $\lambda \in \mathbf{R}$ (which we shall call $\alpha(c)$ ) such that $\Lambda_{c, \lambda}$ has a fixed point in $C_{\text {Group }}(\mathbf{T})$. By point 2 of Proposition 2.1, any fixed point is L-Lipschitz. 
2. Let $U$ be a fixed point of $\Lambda_{c, \lambda}$, and let $M \in$ Mon. Then, there is $\sigma \in$ $A C_{\text {loc }}\left((-\infty, 0], L^{2}(I)\right)$ such that $\sigma_{t} \in M$ on for $t \in(-\infty, 0), \sigma_{0}=M$ and, for all $k \in \mathbf{N}$,

$$
U(M)=\int_{-k}^{0}\left[\mathcal{L}_{c}\left(t, \sigma_{t}, \dot{\sigma}_{t}\right)+\alpha(c)\right] d t+U\left(\sigma_{-k}\right) .
$$

The function $\sigma$ is c-minimal on $(-\infty, 0)$ and solves $(O D E)_{\text {Lag }}$.

Now we introduce the notation of [7] for the Hopf-Lax semigroups, forward $\left(T_{t}^{-}\right)$and backward $\left(T_{-t}^{+}\right)$in time. The signs + and - point, apparently, in the wrong direction; a possible justification is that, when the semigroup goes forward in time, the characteristics go backward, and vice-versa.

Definition. Let $U \in C_{\text {Group }}(\mathbf{T})$, let $M \in L^{2}(I)$ and let $\alpha(c)$ be as in Proposition 2.2 ; for $t \geq 0$, we define

$$
\left(T_{t}^{-} U\right)(M)=\inf \left\{U\left(\gamma_{0}\right)+\int_{0}^{t}\left[\mathcal{L}_{c}\left(s, \gamma_{s}, \dot{\gamma}_{s}\right)+\alpha(c)\right] \mathrm{d} s: \gamma_{t}=M\right\}
$$

and

$$
\left(T_{-t}^{+} U\right)(M)=\sup \left\{U\left(\gamma_{0}\right)-\int_{-t}^{0}\left[\mathcal{L}_{c}\left(s, \gamma_{s}, \dot{\gamma}_{s}\right)+\alpha(c)\right] \mathrm{d} s: \gamma_{-t}=M\right\} .
$$

We note that, by Proposition 2.1, $T_{t}^{-} U$ and $T_{-t}^{+} U$ belong to $C_{\text {Group }}(\mathbf{T})$. By Proposition 2.2, $T_{1}^{-}=\Lambda_{c, \alpha(c)}$ has a fixed point; we cannot say the same for $T_{-1}^{+}$because the choice $\lambda=\alpha(c)$, which yields a fixed point of $T_{1}^{-}$, may not yield a fixed point of $T_{-1}^{+}$; we shall have to wait until Theorem 4.2 below to see that this is actually the case, and that both operators have fixed points.

By point 5 of Proposition 2.1, if $U$ is a fixed point of $T_{1}^{-}$, then, for $t \geq 0, T_{t+1}^{-} U=T_{t}^{-} U$; in other words, the function $\left(T_{t}^{-} U\right)(M)$ defined on $[0,+\infty) \times L^{2}(I)$ can be extended by periodicity to $\mathbf{R} \times L^{2}(I)$. As a final remark, if $M \in M o n$, it follows by Proposition 2.1 that $\left(T_{t}^{-} U\right)(M)$ and $\left(T_{-t}^{+} U\right)(M)$ are a minimum and a maximum respectively.

Definition. We shall say that a function $U \in C_{\text {Group }}(\mathbf{T})$ is $c$-dominated if, for every $m<n \in \mathbf{Z}$ and every $\sigma \in A C\left([m, n], L^{2}(I)\right)$, we have that

$$
U\left(\sigma_{n}\right)-U\left(\sigma_{m}\right) \leq \int_{m}^{n}\left[\mathcal{L}_{c}\left(t, \sigma_{t}, \dot{\sigma}_{t}\right)+\alpha(c)\right] \mathrm{d} t .
$$

We note that there are $c$-dominated functions: for instance, the fixed points of $T_{1}^{-}$, given by Proposition 2.2, are $c$-dominated by formula 2.1.

Definition. If $\sigma \in A C\left([a, b], L^{2}(I)\right)$ and $\sigma_{t} \in$ Mon for $t \in[a, b]$, we shall say that $\sigma \in A C_{m o n}([a, b])$. By point 4) of Proposition 2.1, if $M \in M o n$ there is $\sigma \in A C_{\text {mon }}$ minimal (or maximal) in the definition of $T_{t}^{-} U(M)$ (or of $T_{-t}^{+} U(M)$.).

Definition. Let $U \in C_{\text {Group }}(\mathbf{T})$ be $c$-dominated and let $a<b \in \mathbf{Z} \cup\{ \pm \infty\}$; we say that $\gamma \in A C_{m o n}([a, b])$ is calibrating if, for any $[m, n] \subset[a, b]$ with $m$ and $n$ integers, we have 


$$
U\left(\gamma_{n}\right)-U\left(\gamma_{m}\right)=\int_{m}^{n}\left[\mathcal{L}_{c}\left(t, \gamma_{t}, \dot{\gamma}_{t}\right)+\alpha(c)\right] \mathrm{d} t .
$$

It follows from (2.1) that a calibrating function $\gamma$ is $c$-minimal on $[a, b]$, and thus it satisfies $(O D E)_{\text {Lag }}$.

We state at once a relation between these definitions; it comes, naturally, from [7].

Lemma 2.3. 1. Let $U \in C_{\text {Group }}(\mathbf{T})$; then $U$ is c-dominated iff $U \leq T_{n}^{-} U$ (or iff $\left.T_{-n}^{+} U \leq U\right)$ for all $n \geq 0$.

2. Moreover, $T_{n}^{-}(U)=U$ (or $T_{-n}^{+} U=U$ ) for all $n \in \mathbf{N}$ iff $U$ is c-dominated and, for each $M \in M$ on, there is a calibrating curve $\gamma \in A C_{m o n}((-\infty, 0])$ (or $\gamma \in A C_{m o n}([0,+\infty))$ with $\gamma_{0}=M$.

Proof. Point 1 is a rewording of the definition of $c$-dominated. We prove point 2 ; if $T_{n}^{-} U=U$, then $U$ is $c$-dominated by point 1 ; the existence of a calibrating curve $\gamma$ follows from point 2 of Proposition 2.2. To prove the converse, let $M \in M o n$ and let $\gamma$ be calibrating on $(-\infty, 0]$ with $\gamma_{0}=M$; then,

$$
U(M)-U\left(\gamma_{-1}\right)=U\left(\gamma_{0}\right)-U\left(\gamma_{-1}\right)=\int_{-1}^{0}\left[\mathcal{L}_{c}\left(t, \gamma_{t}, \dot{\gamma}_{t}\right)+\alpha(c)\right] \mathrm{d} t .
$$

By the definition of $T_{1}^{-}$, this means that $\left(T_{1}^{-} U\right)(M) \leq U(M)$; since the opposite inequality holds by point 1 , we have that $\left(T_{1}^{-} U\right)(M)=U(M)$ for all $M \in$ Mon. Since $U$ is continuous and equivariant, and since by [8] any $N \in L^{2}(I)$ can be approximated by $M \circ G_{n}+Z_{n}$ with $M \in M o n, G_{n} \in$ Group and $Z_{n} \in L_{\mathbf{Z}}^{2}$, we have that $\left(T_{1}^{-} U\right)(N)=U(N)$ for all $N \in L^{2}(I)$, and we are done.

The Lagrangian $\mathcal{L}_{c}$ has a Legendre transform $\mathcal{H}_{c}$; an easy calculation shows that

$$
\begin{gathered}
\mathcal{H}_{c}: S^{1} \times L^{2}(I) \times L^{2}(I) \rightarrow \mathbf{R} \\
\mathcal{H}_{c}(t, \sigma, p)=\frac{1}{2}\|c+p\|_{L^{2}(I)}^{2}+\mathcal{V}(t, \sigma)+\mathcal{W}(\sigma) .
\end{gathered}
$$

What we really need are subsolutions of Hamilton-Jacobi; that's why we give the following definition.

Definition. We define $\mathrm{Mon}_{3}$ as the set of monotone functions $\gamma$ on $I$ such that $\gamma(1-) \leq \gamma(0)+3$. Let $U: \mathbf{R} \times L^{2}(I) \rightarrow \mathbf{R}$, and let $M \in$ Mon. We say that $(a, \xi) \in \mathbf{R} \times L^{2}(I)$ is the "lazy differential" of $U$ at $(t, M)$ if there is $K>0$ such that

i) $U(t+h, M+N)-U(t, M) \leq a h+\langle\xi, N\rangle+K\left(|h|^{2}+\|N\|^{2}\right)$ $\forall(h, N) \in \mathbf{R} \times L^{2}(I)$

ii) $U(t+h, M+N)-U(t, M) \geq a h+\langle\xi, N\rangle+o(|h|+\| N||)$

for all $N \in L^{2}(I)$ such that $M+N \in M_{0} n_{3}$.

We set $\left(\partial_{t} U(t, M), \partial_{M} U(t, M)\right):=(a, \xi)$. 
Lemma 2.4. If $U$ is lazily differentiable at $(t, M)$, then the lazy differential $(a, \xi)$ is unique.

Proof. Let $\left(a^{\prime}, \xi^{\prime}\right)$ be another lazy differential; if we set $N=0$ in $\left.\left.i\right), i i\right)$, we get that $a=a^{\prime}$.

If we set $h=0, N=\epsilon \bar{N}$ for $\epsilon>0$, and we subtract $i i$ )

$$
U(t, M+\epsilon \bar{N})-U(t, M) \geq \epsilon\left\langle\xi^{\prime}, \bar{N}\right\rangle+o(\epsilon)
$$

from $i)$

$$
U(t, M+\epsilon \bar{N})-U(t, M) \leq \epsilon\langle\xi, \bar{N}\rangle+K \epsilon^{2}
$$

we get that

$$
\left\langle\xi-\xi^{\prime}, \bar{N}\right\rangle \geq 0
$$

for all $\bar{N}$ such that $M+\epsilon \bar{N} \in$ Mon $_{3}$ for $\epsilon$ positive and small. Exchanging the rôles of $\xi$ and $\xi^{\prime}$, we get that

$$
\left\langle\xi-\xi^{\prime}, \bar{N}\right\rangle=0
$$

for all $\bar{N}$ such that $M+\epsilon \bar{N} \in$ Mon $_{3}$ for $\epsilon$ positive and small. In particular, the formula above holds for $\bar{N}=1_{[c, 1]}$ and $\bar{N}_{1}=1_{[d, 1]}$; subtracting, we get that

$$
\int_{c}^{d}\left(\xi(x)-\xi^{\prime}(x)\right) \mathrm{d} \nu_{0}(x)=0 \quad \forall 0 \leq c<d \leq 1 .
$$

Thus, $\xi=\xi^{\prime}$, as we wanted.

Proposition 2.5. Let $U \in C_{\text {Group }}(\mathbf{T})$. For $t>0$, let us set $\hat{U}(t, M)=$ $\left(T_{t}^{-} U\right)(M)$. For $(t, M) \in(0,+\infty) \times$ Mon, let us suppose that there is a unique curve $\sigma$ such that $\sigma_{t}=M$ and

$$
\hat{U}\left(t, \sigma_{t}\right)-U\left(\sigma_{0}\right)=\int_{0}^{t}\left[\mathcal{L}_{c}\left(s, \sigma_{s}, \dot{\sigma}_{s}\right)+\alpha(c)\right] \mathrm{d} s .
$$

Then, $\hat{U}$ is lazily differentiable at $(t, M)$ and

$$
\partial_{t} \hat{U}(t, M)+\mathcal{H}_{0}\left(t, M, c+\partial_{M} \hat{U}(t, M)\right)=\alpha(c) .
$$

As a partial converse, if $\hat{U}$ is Fréchet differentiable at $(t, M) \in(0,+\infty) \times$ Mon, then there is a unique $\sigma$ minimal in (2.2), which satisfies (2.3) by the statement above.

Proof. The proof is identical to the finite-dimensional one. We begin with the converse.

Let $\hat{U}$ be Fréchet differentiable at $(t, M) \in(0,+\infty) \times$ Mon; by Proposition 2.1, there is a curve $\sigma$ such that (2.2) holds; we want to prove that it is unique. For $N \in L^{2}(I)$, let us set

$$
\tilde{\sigma}_{s}=\sigma_{s}+(N-M) \frac{s}{t} .
$$


Since $\tilde{\sigma}_{t}=N, \tilde{\sigma}_{0}=\sigma_{0}$ and $\sigma$ is minimal, the definition of $\hat{U}$ implies the first inequality below.

$$
\begin{aligned}
\hat{U}( & t, N)-\hat{U}(t, M) \\
\leq & \int_{0}^{t}\left[\mathcal{L}_{c}\left(s, \tilde{\sigma}_{s}, \dot{\tilde{\sigma}}_{s}\right)-\mathcal{L}\left(s, \sigma_{s}, \dot{\sigma}_{s}\right)\right] \mathrm{d} s \leq \int_{0}^{t}\left[\left\langle\dot{\sigma}_{s}-c, \frac{N-M}{t}\right\rangle\right. \\
& \left.-\left\langle\mathcal{V}^{\prime}\left(s, \sigma_{s}\right)+\mathcal{W}^{\prime}\left(\sigma_{s}\right), \frac{(N-M) s}{t}\right\rangle\right] \mathrm{d} s+K\|N-M\|^{2} \\
= & \left\langle\dot{\sigma}_{t}-c, N-M\right\rangle+K\|N-M\|^{2} .
\end{aligned}
$$

The second inequality above comes from a Taylor development of $\mathcal{L}_{c}$, and from the fact that the second derivatives of $V$ and $W$ are bounded; the equality comes from an integration by parts and the fact that $\sigma$, by point 4 ) of Proposition 2.1, solves $(O D E)_{\text {Lag }}$.

If $\hat{U}$ is Fréchet differentiable at $(t, M)$, the last formula implies that

$$
\partial_{M} \hat{U}(t, M)=\left(\dot{\sigma}_{t}-c\right) \text {. }
$$

Since $\sigma$ satisfies (2.2), it is calibrating, and thus it solves $(O D E)_{\text {Lag }}$; we have just seen that its final speed at $t$ satisfies the formula above; since the existence and uniqueness theorem holds for $(O D E)_{L a g}$, we get that the minimizer at $(t, M)$ is unique. It remains to prove that (2.3) holds; since we have just shown that the minimizer $\sigma$ is unique, this follows from the direct statement, which we presently prove.

Let us suppose that $(t, M) \in(0,+\infty) \times$ Mon, and let the minimum in (2.2) be attained on a unique $\sigma$. We want to prove that $\hat{U}$ is lazily differentiable and satisfies (2.3) at $(t, M)$. For $h \in \mathbf{R}$ and $N \in L^{2}(I)$, we set

$$
\tilde{\sigma}_{s}=\sigma_{s}+\frac{N-M}{t+h} s-\left(\sigma_{t+h}-\sigma_{t}\right) \frac{s}{t+h}
$$

and we see that $\tilde{\sigma}_{t+h}=N$ while $\tilde{\sigma}_{0}=\sigma_{0}$. We get as above that

$$
\begin{aligned}
& \hat{U}(t+h, N)-\hat{U}(t, M) \\
& \quad \leq \int_{0}^{t+h}\left[\mathcal{L}_{c}\left(s, \tilde{\sigma}_{s}, \dot{\tilde{\sigma}}_{s}\right)+\alpha(c)\right] \mathrm{d} s-\int_{0}^{t}\left[\mathcal{L}_{c}\left(s, \sigma_{s}, \dot{\sigma}_{s}\right)+\alpha(c)\right] \mathrm{d} s \\
& \quad=\int_{t}^{t+h}\left[\mathcal{L}_{c}\left(s, \sigma_{s}, \dot{\sigma}_{s}\right)+\alpha(c)\right] \mathrm{d} s+\int_{0}^{t+h}\left[\mathcal{L}_{c}\left(s, \tilde{\sigma}_{s}, \dot{\tilde{\sigma}}_{s}\right)-\mathcal{L}_{c}\left(s, \sigma_{s}, \dot{\sigma}_{s}\right)\right] \mathrm{d} s .
\end{aligned}
$$

We also note that, since $\left\|V^{\prime}\right\|_{\infty}+\left\|W^{\prime}\right\|_{\infty} \leq K$, we have $\left\|\mathcal{V}^{\prime}\right\|+\left\|\mathcal{W}^{\prime}\right\| \leq$ $K$; we recall that $\|\cdot\|$ denotes the norm on $L^{2}(I)$. Since $\sigma_{t}$ solves $(O D E)_{L a g}$, this yields that $\left\|\ddot{\sigma}_{t}\right\| \leq K$; by a Taylor development, this implies that

$$
\left\|\frac{\sigma_{t+h}-\sigma_{t}}{h}-\dot{\sigma}_{t}\right\| \leq K|h| .
$$

The last two formulas and a Taylor development imply the first inequality below; the equality comes from an integration by parts; the last inequality comes again from (2.6). 


$$
\begin{aligned}
\hat{U}(t & +h, N)-\hat{U}(t, M) \leq h\left[\mathcal{L}_{c}\left(t, \sigma_{t}, \dot{\sigma}_{t}\right)+\alpha(c)\right] \\
& +\int_{0}^{t+h}\left[\left\langle\dot{\sigma}_{s}-c, \frac{(N-M)-\left(\sigma_{t+h}-\sigma_{t}\right)}{t+h}\right\rangle\right. \\
& \left.-\left\langle\mathcal{V}^{\prime}\left(s, \sigma_{s}\right)+\mathcal{W}^{\prime}\left(\sigma_{s}\right) \frac{\left[(N-M)-\left(\sigma_{t+h}-\sigma_{t}\right)\right] s}{t+h}\right\rangle\right] \mathrm{d} s \\
& +K\left(h^{2}+\|N-M\|^{2}\right)=h\left[\mathcal{L}_{c}\left(t, \sigma_{t}, \dot{\sigma}_{t}\right)+\alpha(c)\right] \\
& +\left\langle\dot{\sigma}_{t+h}-c,-\sigma_{t+h}+\sigma_{t}+(N-M)\right\rangle+K\left(h^{2}+\|N-M\|^{2}\right) \\
\leq & h\left[\mathcal{L}_{c}\left(t, \sigma_{t}, \dot{\sigma}_{t}\right)+\alpha(c)\right] \\
& -h\left\langle\dot{\sigma}_{t}-c, \dot{\sigma}_{t}\right\rangle+\left\langle\dot{\sigma}_{t}-c, N-M\right\rangle+2 K\left(h^{2}+\|N-M\|^{2}\right) .
\end{aligned}
$$

Since

$$
-\mathcal{H}_{0}\left(t, \sigma_{t}, \dot{\sigma}_{t}\right)=\mathcal{L}_{c}\left(t, \sigma_{t}, \dot{\sigma}_{t}\right)-\left\langle\dot{\sigma}_{t}-c, \dot{\sigma}_{t}\right\rangle,
$$

the last formula implies that

$$
\begin{aligned}
& \hat{U}(t+h, N)-\hat{U}(t, M) \leq-h\left[\mathcal{H}_{0}\left(t, \sigma_{t}, \dot{\sigma}_{t}\right)-\alpha(c)\right] \\
& \quad+\left\langle\dot{\sigma}_{t}-c, N-M\right\rangle+2 K\left(h^{2}+\|N-M\|^{2}\right) .
\end{aligned}
$$

To prove differentiability and (2.3), we need an inequality opposite to (2.7). We let $\sigma$ be as above, the minimizing curve for $\hat{U}(t, M)$; by hypothesis, $\sigma$ is unique. We note that point 4) of Proposition 2.1 holds for $\gamma(0) \in \mathrm{Mon}_{3}$, with the same proof. In other words, if $N \in M_{\text {on }}$ we can find $\sigma^{h, N}$ minimal for $\hat{U}(t+h, N)$ such that $\sigma_{t+h}^{h, N}=N$; moreover, $\sigma_{s}^{h, N} \in$ Mon $_{3}$ for $0 \leq s \leq t+h$. We set

$$
\hat{\sigma}_{s}=\sigma_{s}^{h, N}+\frac{M-N}{t} s+\frac{\sigma_{t+h}^{h, N}-\sigma_{t}^{h, N}}{t} s
$$

and we see that $\hat{\sigma}_{t}=M, \hat{\sigma}_{0}=\sigma_{0}^{h, N}$. With the same calculations of $(2.7)$, we get that

$$
\begin{aligned}
\hat{U}(t, M) & -\hat{U}(t+h, N) \leq \int_{0}^{t}\left[\mathcal{L}_{c}\left(s, \hat{\sigma}_{s}, \dot{\hat{\sigma}}_{s}\right)+\alpha(c)\right] \mathrm{d} s \\
& -\int_{0}^{t+h}\left[\mathcal{L}_{c}\left(s, \sigma_{s}^{h, N}, \dot{\sigma}_{s}^{h, N}\right)+\alpha(c)\right] \mathrm{d} s \leq-\left\langle\dot{\sigma}_{t}^{h, N}-c, N-M\right\rangle \\
& +h\left[\mathcal{H}_{0}\left(t, N, \dot{\sigma}_{t+h}^{h, N}\right)-\alpha(c)\right]+K\left(h^{2}+\|M-N\|^{2}\right) .
\end{aligned}
$$

We forego the easy proof $([5])$ that, if $(t+h, N)$ belongs to a ball centered in $(t, M)$, we have a uniform bound

$$
\int_{0}^{t}\left\|\dot{\sigma}_{s}^{h, N}\right\|^{2} \mathrm{~d} s \leq C_{1} .
$$

In particular, $\sigma_{s}^{h, N}$ is uniformly $\frac{1}{2}$-Hölder for $|h| \leq 1$ if $N \in M_{3}$ and $\| M-$ $N \| \leq 1$.

We assert that the uniform Holderianity of $\sigma_{s}^{h, N}$ implies the following: if $N \in \mathrm{Mon}_{3}$ and $|h|+\|M-N\|<\delta$, then

$$
\left\|\sigma^{h, N}-\sigma\right\|_{C^{0}\left([0, t], M_{0}\right)}<\epsilon(\delta) \quad \text { with } \epsilon(\delta) \rightarrow 0 \quad \text { as } \quad \delta \rightarrow 0 .
$$


It suffices to show that, if $\left(h_{k}, N_{k}\right) \rightarrow(0, M)$ in $\mathbf{R} \times M_{\text {on }}$, then, up to subsequences, $\sigma^{h_{k}, N_{k}} \rightarrow \sigma$ in $C^{0}\left([0, t], M_{o n}\right)$. We show this fact.

Since $\sigma^{h_{k}, N_{k}}:[0, t] \rightarrow \operatorname{Mon}_{3}$ and Mon $_{3}$ is locally compact, we can use (2.9) and Ascoli-Arzelà as in [5] to get that, up to subsequences, $\sigma^{h_{k}, N_{k}} \rightarrow \sigma^{1}$ in $C^{0}\left([0, t]\right.$, Mon $\left._{3}\right)$. Since $\sigma^{h_{k}, N_{k}}$ minimizes in (2.1), we easily see [5] that $\sigma^{1}$ minimizes $(2.1)$ at $(t, M)$. By our hypotheses, $\sigma$ is the only minimizer; this yields that $\sigma^{1}=\sigma$. In other words, $\sigma^{h, N} \rightarrow \sigma$ in $C^{0}\left([0, t]\right.$, Mon $\left._{3}\right)$ as $(t+h, N) \rightarrow(t, M)$; since $\sigma^{h, N}$ satisfies $(O D E)_{L a g}$, it follows that $\sigma^{h, N} \rightarrow \sigma$ in $C^{2}\left([0, t]\right.$, Mon $\left._{3}\right)$. This fact and (2.8) imply that, for $N \in M_{o n}$,

$$
\begin{aligned}
\hat{U}(t, M)-\hat{U}(t+h, N) \leq & -\left\langle\dot{\sigma}_{t}-c, N-M\right\rangle+h\left[\mathcal{H}_{0}\left(t, M, \dot{\sigma}_{t}\right)-\alpha(c)\right] \\
& +\epsilon(\|M-N\|+|h|) \cdot(\|M-N\|+|h|)
\end{aligned}
$$

where $\epsilon(\gamma) \rightarrow 0$ as $\gamma \rightarrow 0$.

The last formula, together with (2.7), implies that $\hat{U}$ is lazily differentiable and that

$$
\partial_{M} \hat{U}(t, M)=\left(\dot{\sigma}_{t}-c\right), \quad \partial_{t} \hat{U}(t, M)=-\mathcal{H}_{0}\left(t, \sigma_{t}, \dot{\sigma}_{t}\right)+\alpha(c) .
$$

Since $\sigma_{t}=M,(2.3)$ holds.

Lemma 2.6. There is $K \geq 0$ such that, for any $U \in C_{\mathrm{Group}}(\mathbf{T})$, the function $T_{1}^{-} U$ is $K$-quasiconcave. In other words, there is $K \geq 0$ such that the map $\Phi_{K}$

$$
\Phi_{K}: L^{2}(I) \rightarrow \mathbf{R}, \quad \Phi_{K}: M \rightarrow\left(T_{1}^{-} U\right)(M)-\frac{K}{2}\|M\|^{2}
$$

is concave.

Proof. We define a Lagrangian on $S^{1} \times\left(S^{1}\right)^{n} \times \mathbf{R}^{n}$ by

$$
L_{n, c}(t, q, \dot{q})=\frac{1}{n} \sum_{i=1}^{n}\left(\frac{1}{2}\left|\dot{q}_{i}\right|^{2}-c \dot{q}_{i}\right)-\frac{1}{n} \sum_{i=1}^{n} V\left(t, q_{i}\right)-\frac{1}{2 n^{2}} \sum_{i, j=1}^{n} W\left(q_{i}-q_{j}\right)
$$

where $q=\left(q_{1}, \ldots, q_{n}\right)$. This is the Lagrangian for the Vlasov equation with $n$ particles, each of mass $\frac{1}{n}$; its value function is

$$
\begin{gathered}
\hat{u}_{n}(x)=\min \left\{\int_{0}^{1}\left[L_{n, c}(t, q, \dot{q})+\alpha(c)\right] \mathrm{d} t+U\left(D_{n} q(0)\right): q \in A C\left([0,1], \mathbf{R}^{n}\right),\right. \\
q(1)=x\}
\end{gathered}
$$

where the operator $D_{n}$ has been defined at the end of Sect. 1, and $x=$ $\left(x_{1}, \ldots, x_{n}\right)$.

Since $L_{n, c}$ is a finite-dimensional Lagrangian, the minimum above is attained by Tonelli's theorem. Let $q$ be minimal in the definition of $\hat{u}_{n}(x)$; for $h \in \mathbf{R}^{n}$, we set

$$
q^{ \pm h}=q(t) \pm h t
$$


Formula (2.10) implies the first inequality below.

$$
\begin{aligned}
& \hat{u}_{n}(x+h)+\hat{u}_{n}(x-h)-2 \hat{u}_{n}(x) \leq \int_{0}^{1}\left[L_{n, c}\left(t, q^{h}, \dot{q}^{h}\right)+L_{n, c}\left(t, q^{-h}, \dot{q}^{-h}\right)\right. \\
& \left.-2 L_{n, c}(t, q, \dot{q})\right] \mathrm{d} t=\int_{0}^{1}\left\{\frac{1}{n}|h|^{2}-\frac{1}{2 n} \sum_{i=1}^{n}\left[V^{\prime \prime}\left(t, q_{i}(t)+\theta_{i}^{+} h_{i}(t-1)\right)\right.\right. \\
& \left.+V^{\prime \prime}\left(t, q_{i}(t)-\theta_{i}^{-} h_{i}(t-1)\right)\right] h_{i}^{2}-\frac{1}{4 n^{2}} \sum_{i, j=1}^{n}\left[W ^ { \prime \prime } \left(q_{i}(t)-q_{j}(t)\right.\right. \\
& \left.\left.+\theta_{i, j}^{+}\left(h_{i}-h_{j}\right)(t-1)\right)+W^{\prime \prime}\left(q_{i}(t)-q_{j}(t)-\theta_{i, j}^{-}\left(h_{i}-h_{j}\right)(t-1)\right)\right]\left(h_{i}-h_{j}\right)^{2} .
\end{aligned}
$$

The equality above comes from a second order Taylor development (the constants $\theta_{i}^{ \pm}$and $\theta_{i, j}^{ \pm}$belong to $(0,1)$ and depend on $\left.t\right)$; since

$$
\left|V^{\prime \prime}(t, x)\right| \leq C_{1}, \quad\left|W^{\prime \prime}(x)\right| \leq C_{1}, \quad \text { and } \quad\left(h_{i}-h_{j}\right)^{2} \leq 2 h_{i}^{2}+2 h_{j}^{2},
$$

we get that

$$
\begin{aligned}
& \hat{u}_{n}(x+h)+\hat{u}_{n}(x-h)-2 \hat{u}_{n}(x) \\
& \quad \leq \frac{1}{n}|h|^{2}+\frac{1}{2 n} \sum_{i=1}^{n} 2 C_{1}\left(h_{i}\right)^{2}+\frac{1}{4 n^{2}} \sum_{i, j=1}^{n} 4 C_{1}\left(h_{i}^{2}+h_{j}^{2}\right)=\frac{K}{n}|h|^{2}
\end{aligned}
$$

where we have denoted by $|\cdot|$ the euclidean norm in $\mathbf{R}^{n}$. It is well-known that the formula above implies that the function from $\mathbf{R}^{n}$ to $\mathbf{R}$

$$
: x \rightarrow \hat{u}_{n}(x)-\frac{1}{2} \frac{K}{n}|x|^{2}
$$

is concave. By the definition of the operator $D_{n}: \mathbf{R}^{n} \rightarrow L^{2}(I)$, we have that $\frac{1}{\sqrt{n}}|q|=|| D_{n} q \|$; thus, the formula above says that the function from $L^{2}(I)$ to $\mathbf{R}$

$$
: M \rightarrow \hat{u}_{n}\left(P_{n} M\right)-\frac{K}{2}\left\|P_{n} M\right\|^{2}
$$

is concave. The thesis follows from this and from the fact, proven in [5], that, if $M \in L^{2}(I)$, then

$$
\hat{u}_{n}\left(P_{n} M\right) \rightarrow\left(T_{1}^{-} U\right)(M) \quad \text { as } \quad n \rightarrow+\infty .
$$

Definition. Let $U \in C_{\text {Group }}(\mathbf{T})$ be $c$-dominated; we define $A_{U}$ as the set of the $M \in M o n$ for which there is $\gamma \in A C_{\text {mon }}([-1,1])$ with $\gamma_{0}=M$ and

$$
U\left(\gamma_{1}\right)-U\left(\gamma_{-1}\right)=\int_{-1}^{1}\left[\mathcal{L}_{c}\left(s, \gamma_{s}, \dot{\gamma}_{s}\right)+\alpha(c)\right] \mathrm{d} s .
$$

Theorem 2.7. There is a constant $A>0$ such that, if $U$ is c-dominated, then the following holds.

1) $A_{U}$ is closed in Mon.

2) If $M \in A_{U}$, then $U(M)=\left(T_{1}^{-} U\right)(M)=\left(T_{-1}^{+} U\right)(M)$.

3) If $\gamma$ is as in (2.11) and $\gamma_{0}=M \in A_{U}$, then $\left.\gamma\right|_{[-1,0]}$ is the unique curve on which the inf in the definition of $\left(T_{1}^{-} U\right)(M)$ is attained; analogously, $\left.\gamma\right|_{[0,1]}$ is the unique curve on which the $\sup$ in the definition of $\left(T_{-1}^{+} U\right)(M)$ is attained. 
4) Let us call $\gamma^{M}$ the curve which satisfies (2.11) and $\gamma_{0}^{M}=M$; we recall that, by point 3), $\gamma^{M}$ is unique. Then, the map : $M \rightarrow \dot{\gamma}_{0}^{M}$ is continuous.

5) $U$ is Fréchet differentiable at $M \in A_{U}$, and $d_{M} U=\dot{\gamma}_{0}^{M}-c$. Moreover, the map

$$
: A_{U} \rightarrow L^{2}(I) \times L^{2}(I), \quad: M \rightarrow\left(M, d_{M} U\right)
$$

is Lipschitz with Lipschitz inverse.

Proof. We only sketch the proof, which is identical to theorem 4.5.5 of [7]. We begin with point 1). Let $M^{n} \in A_{U}$ and let $M^{n} \rightarrow M$ in $L^{2}(I)$; let $\gamma^{n} \in$ $A C_{\text {mon }}([-1,1])$ be a curve satisfying $(2.11)$ with $\gamma_{0}^{n}=M^{n}$. We shall prove that $\gamma^{n}$ converges to a curve $\gamma$ which satisfies (2.11) and such that $\gamma_{0}=M$.

Since $\gamma^{n}$ is calibrating, it is $c$-minimal; this implies in a standard way (see Lemma 3.4 below for a proof) that there is $C_{1}>0$ such that

$$
\sup _{t \in(-1,1)}\left\|\dot{\gamma}_{t}^{n}\right\| \leq C_{1} \quad \forall n \in \mathbf{N}
$$

As a consequence, $\gamma^{n}:[-1,1] \rightarrow$ Mon is equilipschitz and, since $\gamma_{0}^{n}=M^{n}$ is bounded, $\gamma^{n}$ is equibounded too. Since Mon is a locally compact subset of $L^{2}(I)$, we get by Ascoli-Arzelà that, up to subsequences, $\gamma^{n} \rightarrow \gamma$ in $C^{0}\left([-1,1], L^{2}(I)\right)$, and that $\gamma_{0}=M$. Using the fact that $\gamma_{n}$ solves $(O D E)_{\text {Lag }}$, we see that $\ddot{\gamma}_{n} \rightarrow \ddot{\gamma}$ in $C^{0}\left([-1,1], L^{2}(I)\right)$; by the interpolation inequalities, $\gamma^{n} \rightarrow \gamma$ in $C^{2}\left([-1,1], L^{2}(I)\right)$. Now, the action functional is continuous under convergence in $C^{1}$; together with the fact that $U$ is continuous and that $\gamma^{n}$ satisfies (2.11), this implies that

$$
U\left(\gamma_{1}\right)-U\left(\gamma_{-1}\right)=\int_{-1}^{1}\left[\mathcal{L}_{c}\left(t, \gamma_{t}, \dot{\gamma}_{t}\right)+\alpha(c)\right] \mathrm{d} t
$$

proving point 1 ). We prove point 2). Since $U$ is $c$-dominated, point 1 ) of Lemma 2.3 implies that

$$
U(M) \leq\left(T_{1}^{-} U\right)(M) \quad \text { and } \quad\left(T_{-1}^{+} U\right)(M) \leq U(M) \text { for all } M \in L^{2}(I) .
$$

Let now $M \in A_{U}$ and let $\gamma$ with $\gamma_{0}=M$ satisfy (2.11). We re-write $(2.11)$ as

$$
\begin{aligned}
& U\left(\gamma_{1}\right)-U(M)+U(M)-U\left(\gamma_{-1}\right)=\int_{-1}^{0}\left[\mathcal{L}_{c}\left(s, \gamma_{s}, \dot{\gamma}_{s}\right)+\alpha(c)\right] \mathrm{d} s \\
& \quad+\int_{0}^{1}\left[\mathcal{L}_{c}\left(s, \gamma_{s}, \dot{\gamma}_{s}\right)+\alpha(c)\right] \mathrm{d} s .
\end{aligned}
$$

Since $U$ is $c$-dominated, we also have that

$$
\left\{\begin{array}{l}
U\left(\gamma_{1}\right)-U(M)=U\left(\gamma_{1}\right)-U\left(\gamma_{0}\right) \leq \int_{0}^{1}\left[\mathcal{L}_{c}\left(s, \gamma_{s}, \dot{\gamma}_{s}\right)+\alpha(c)\right] \mathrm{d} s \\
U(M)-U\left(\gamma_{-1}\right)=U\left(\gamma_{0}\right)-U\left(\gamma_{-1}\right) \leq \int_{-1}^{0}\left[\mathcal{L}_{c}\left(s, \gamma_{s}, \dot{\gamma}_{s}\right)+\alpha(c)\right] \mathrm{d} s
\end{array}\right.
$$


From the last two formulas, we get that

$$
\begin{gathered}
U(M)-U\left(\gamma_{-1}\right)=\int_{-1}^{0}\left[\mathcal{L}_{c}\left(s, \gamma_{s}, \dot{\gamma}_{s}\right)+\alpha(c)\right] \mathrm{d} s \\
U\left(\gamma_{1}\right)-U(M)=\int_{0}^{1}\left[\mathcal{L}_{c}\left(s, \gamma_{s}, \dot{\gamma}_{s}\right)+\alpha(c)\right] \mathrm{d} s .
\end{gathered}
$$

By the definitions of $\left(T_{1}^{-} U\right)(M)$ and $\left(T_{-1}^{+} U\right)(M)$, the two formulas above imply respectively that, if $M \in A_{U}$,

$$
\left(T_{1}^{-} U\right)(M) \leq U(M) \quad \text { and } \quad U(M) \leq\left(T_{-1}^{+} U\right)(M) .
$$

This and (2.12) prove point 2).

We prove point 3). By point 2), if $M \in A_{U}$, then $\left(T_{1}^{-} U\right)(M)=U(M)$; thus, it suffices to prove that any curve $\tilde{\gamma}$ with $\tilde{\gamma}_{0}=M$ and

$$
\left(T_{1}^{-} U\right)\left(\tilde{\gamma}_{0}\right)-U\left(\tilde{\gamma}_{-1}\right)=U\left(\tilde{\gamma}_{0}\right)-U\left(\tilde{\gamma}_{-1}\right)=\int_{-1}^{0}\left[\mathcal{L}_{c}\left(t, \tilde{\gamma}_{t}, \dot{\tilde{\gamma}}_{t}\right)\right] \mathrm{d} t
$$

coincides with $\gamma$.

Let us suppose by contradiction that $\tilde{\gamma} \neq \gamma$ on $[-1,0]$; we define

$$
\hat{\gamma}_{t}= \begin{cases}\tilde{\gamma}_{t} & t \in[-1,0] \\ \gamma_{t} & t \in[0,1]\end{cases}
$$

By (2.14) and the second formula of (2.13), it follows easily that (2.11) holds for $\hat{\gamma}$. We have seen that this implies that $\hat{\gamma}$ is $c$-minimal on $[-1,1]$; in particular, it satisfies $(O D E)_{\text {Lag }}$. Now $\gamma$ satisfies $(O D E)_{\text {Lag }}$ for the same reason; since $\hat{\gamma}=\gamma$ on $[0,1]$, we have a contradiction with the existence and uniqueness theorem.

We prove point 4). Let $M_{n} \in A_{U}$, and let $M_{n} \rightarrow M$ in $L^{2}(I)$; point 1) implies that $M \in A_{U}$. Let $\gamma^{M_{n}}$ satisfy (2.11) with $\gamma_{0}^{M_{n}}=M_{n}$; we see as in the proof of point 1$)$ that the sequence $\gamma^{M_{n}} \in A C_{m o n}(-1,1)$ has a subsequence converging to a limit $\gamma$ in $C^{2}\left([-1,1], L^{2}(I)\right)$. As a consequence, $\gamma$ satisfies (2.11) and $\gamma_{0}=M$. By the uniqueness of point 3$)$, this implies that $\gamma^{M_{n}} \rightarrow \gamma^{M}$ in $C^{2}\left((-1,1), L^{2}(I)\right)$, proving 4$)$.

We prove point 5$)$. Let $M \in A_{U}$; the inequality below is point 1) Lemma 2.3 ; the equality, point 2$)$ of the present theorem.

$$
U(N) \leq\left(T_{1}^{-} U\right)(N) \quad \forall N \in L^{2}(I) \quad \text { and } \quad U(M)=\left(T_{1}^{-} U\right)(M) .
$$

This implies the first inequality and the equality below; in the proof of Proposition 2.4, we got (2.4), i.e. the second inequality below.

$$
\begin{aligned}
U(N) & \leq\left(T_{1}^{-} U\right)(N) \leq\left(T_{1}^{-} U\right)(M)+\left\langle\dot{\gamma}_{0}^{M}-c, N-M\right\rangle+K\|N-M\|^{2} \\
& =U(M)+\left\langle\dot{\gamma}_{0}^{M}-c, N-M\right\rangle+K\|N-M\|^{2} \quad \forall N \in L^{2}(I) .
\end{aligned}
$$

Applying the same argument to $T_{-1}^{+}$with time reversed, we get that

$$
U(N) \geq U(M)+\left\langle\dot{\gamma}_{0}^{M}-c, N-M\right\rangle-K\|N-M\|^{2} \quad \forall N \in L^{2}(I) .
$$

Now a general fact (Proposition 4.5.3 of [7]) implies that, if the two inequalities above hold, then $U$ is Fréchet differentiable at any point of $A_{U}$, with $d_{M} U=\dot{\gamma}_{0}^{M}-c$. Moreover, the map $: M \rightarrow d_{M} U$ is Lipschitz. 


\section{The minimal measures}

Definition. Let $M, N \in M o n$; we say that $M \simeq N$ if $M-N \equiv z \in \mathbf{Z}$. We denote by $\mathrm{Mon}_{\mathbf{Z}}$ the space of equivalence classes; it is easy to see that $\mathrm{Mon}_{\mathbf{Z}}$ is compact for the topology it inherits from Mon (or from $L^{2}(I)$, which is the same.) We shall denote by $[[M]]$ the equivalence class of $M$ in $M o n_{\mathbf{Z}}$ : we use the double brackets to avoid confusion with the equivalence class of $M$ in $\mathbf{S}$, which we denoted by $[\mathrm{M}]$. We denote by $\Pi$ the natural projection of $M o n$ into $M_{\mathrm{Z}}$. In the following, we shall work mostly on Mon, though we shall turn to $M n_{\mathbf{Z}}$ in all situations in which we need compactness.

We let $B_{R}$ be the closed ball of radius $R$ in $L^{2}(I)$, with the weak topology; we endow

$$
F_{R}:=S^{1} \times \operatorname{Mon}_{\mathbf{Z}} \times B_{R}
$$

with the product topology. We see that $F_{R}$, being the product of compact sets, is compact; moreover, it is a metric space.

Let $\psi_{s}(t, M, v)$ be the flow of $(O D E)_{\text {Lag }}$; in other words,

$$
\psi_{s}: \mathbf{R} \times L^{2}(I) \times L^{2}(I) \rightarrow \mathbf{R} \times L^{2}(I) \times L^{2}(I), \quad \psi_{s}(t, M, v)=\left(t+s, \gamma_{t+s}, \dot{\gamma}_{t+s}\right)
$$

where $\gamma_{\tau}$ solves

$$
\left\{\begin{array}{l}
\ddot{\gamma}_{\tau}=-\mathcal{V}^{\prime}\left(\tau, \gamma_{\tau}\right)-\mathcal{W}^{\prime}\left(\gamma_{\tau}\right) \text { for } \quad \tau \in \mathbf{R} \\
\gamma_{t}=M \\
\dot{\gamma}_{t}=v
\end{array}\right.
$$

We want to restrict this flow to a compact subset $K_{R}$ of $F_{R}$.

Definition. We define the set $K_{R} \subset F_{R}$ in the following way. Let $(t,[[M]], v) \in$ $F_{R}$ and let $\psi_{s}(t, M, v)=\left(t+s, \gamma_{t+s}, \dot{\gamma}_{t+s}\right)$; if $\gamma_{\tau} \in M o n$ and $\left\|\dot{\gamma}_{\tau}\right\| \leq R$ for all $\tau \in \mathbf{R}$, we say that $(t,[[M]], v) \in K_{R}$. Note that, since $\psi_{s}(t, M+k, v)=$ $\psi_{s}(t, M, v)+(0, k, 0)$, the condition just stated does not depend on the choice of the representative $M$.

We are going to see below that, for $R$ large, $K_{R} \neq \emptyset$; meanwhile, we prove the following.

Lemma 3.1. $K_{R}$ is compact in $F_{R}$.

Proof. Since we saw above that $F_{R}$ is compact, it suffices to prove that $K_{R}$ is closed in $F_{R}$. Thus, let $\left(t_{n},\left[\left[M_{n}\right]\right], v_{n}\right) \in K_{R}$, and let $\left(t_{n},\left[\left[M_{n}\right]\right], v_{n}\right) \rightarrow$ $(t,[[M]], v)$; we must prove that $(t,[[M]], v) \in K_{R}$.

First of all, up to adding integers, we can suppose that $M_{n} \rightarrow M$ in Mon. By the definition of $K_{R}$, we can find a solution $\gamma^{n}$ of $(O D E)_{\text {Lag }}$ such that $\left(\gamma_{t_{n}}^{n}, \dot{\gamma}_{t_{n}}^{n}\right)=\left(M_{n}, v_{n}\right)$ and $\gamma_{s}^{n} \in M o n,\left\|\dot{\gamma}_{s}^{n}\right\| \leq R$ for all $s \in \mathbf{R}$. As a consequence, $\gamma^{n}: \mathbf{R} \rightarrow$ Mon is $R$-Lipschitz for all $n$; since $\left(t_{n}, \gamma_{t_{n}}^{n}\right) \rightarrow(t, M)$, we get that $\gamma_{n}$ is locally bounded. Now, bounded sets of Mon are relatively compact, and we can apply Ascoli-Arzelà and get that, up to subsequences, $\gamma^{n} \rightarrow \gamma$ in $C_{l o c}^{0}(\mathbf{R}, M o n)$.

Clearly, $\gamma_{s} \in$ Mon for all $s$; indeed, it is the $L^{2}(I)$-limit of $\gamma_{s}^{n} \in M o n$. 
We note that $\sup _{s \in \mathbf{R}}\left\|\dot{\gamma}_{s}\right\|$ is l. s. c. for the $C_{l o c}^{0}(\mathbf{R}$, Mon $)$ topology; indeed, if $\|\psi\| \leq 1$, we have that

$$
\left|\left\langle\dot{\gamma}_{s}, \psi\right\rangle\right|=\left|\frac{\mathrm{d}}{\mathrm{d} s}\left\langle\gamma_{s}, \psi\right\rangle\right| \leq \sup _{s}\left|\frac{\mathrm{d}}{\mathrm{d} s}\left\langle\gamma_{s}, \psi\right\rangle\right| \leq \liminf _{n \rightarrow+\infty} \sup _{\tau \in \mathbf{R}}\left|\frac{\mathrm{d}}{\mathrm{d} \tau}\left\langle\gamma_{\tau}^{n}, \psi\right\rangle\right| \leq R .
$$

The second inequality above comes from the well-known fact that, in dimension 1, the sup norm of the derivative is 1 . s. c. for uniform convergence; the third one comes from the fact that $\left(\tau, \gamma_{\tau}^{n}, \dot{\gamma}_{\tau}^{n}\right) \in K_{R}$. Since $\psi$ is arbitrary in $B_{1}$ and $s$ is arbitrary in $\mathbf{R}$, the formula above implies

$$
\sup _{s \in \mathbf{R}}\left\|\dot{\gamma}_{s}\right\| \leq R
$$

We prove that $\gamma$ is an orbit with $\gamma_{t}=M$ and $\dot{\gamma}_{t}=v$. Since $\gamma^{n} \rightarrow$ $\gamma$ in $C_{l o c}^{0}\left(\mathbf{R}, L^{2}(I)\right)$ and $\gamma^{n}$ satisfies $(O D E)_{L a g}$, it follows that $\ddot{\gamma}^{n} \rightarrow \ddot{\gamma}$ in $C_{l o c}^{0}\left(\mathbf{R}, L^{2}(I)\right)$. By the usual interpolation inequalities, we get that $\dot{\gamma}^{n} \rightarrow \dot{\gamma}$ in $C_{\text {loc }}^{0}\left(\mathbf{R}, L^{2}(I)\right)$, which implies that $\dot{\gamma}_{t}=v$. Finally, since $\gamma_{n} \rightarrow \gamma$ in $C^{2}$, taking limits in $(O D E)_{\text {Lag }}$, we get that $\gamma$ solves this equation.

Definition. We define $\mathcal{M}_{1}^{R}$ as the set of the probability measures on $K_{R}$, invariant by the Euler-Lagrange flow of $\mathcal{L}$. We note that $\mathcal{M}_{1}^{R}$ is not empty, if $R$ is large enough; to show this, we recall that $W^{\prime}(0)=0$, because $W$ is even; thus, if $q(t)$ is an orbit of the one-particle Lagrangian

$$
L: S^{1} \times S^{1} \times \mathbf{R} \rightarrow \mathbf{R}, \quad L(t, q, \dot{q})=\frac{1}{2}|\dot{q}|^{2}-V(t, q),
$$

then $D_{1} q(t)$ is an orbit of $\mathcal{L}$; the operator $D_{1}$ has been defined at the end of Sect. 1. As a consequence, if $R$ is large enough, $\mathcal{M}_{1}^{R}$ contains the measures induced by $\left(t,\left[\left[D_{1} q(t)\right]\right], \dot{q}(t)\right)$, where $q$ is a periodic orbit of $L$.

We endow $\mathcal{M}_{1}^{R}$ with the weak* topology; since $K_{R}$ is a compact metric space, we get that $\mathcal{M}_{1}^{R}$ is a compact metric space too.

We also define

$$
I_{c}: \mathcal{M}_{1}^{R} \rightarrow \mathbf{R}, \quad I_{c}(\mu)=\int_{K_{R}} \mathcal{L}_{c}(t, \sigma, v) \mathrm{d} \mu(t,[[\sigma]], v) .
$$

Lemma 3.2. The functional $I_{c}$ on $\mathcal{M}_{1}^{R}$ is lower semicontinuous.

Proof. We note that

$$
\mathcal{L}_{c}(t, \sigma, v)=\operatorname{Cin}(v)-\operatorname{Hom}(v)-P(t, \sigma)
$$

where

$$
\operatorname{Cin}(v)=\frac{1}{2}\|v\|^{2}, \quad \operatorname{Hom}(v)=\langle c, v\rangle, \quad P(t, \sigma)=\mathcal{V}(t, \sigma)+\mathcal{W}(t, \sigma) .
$$

Since $V$ and $W$ are Lipschitz, and the topology on $M^{\circ} n_{\mathbf{Z}}$ is the one induced by $L^{2}(I)$, it is immediate that

$$
P: K_{R} \rightarrow \mathbf{R}
$$

is continuous. By the definition of the weak* topology on $\mathcal{M}_{1}^{R}$, this implies that the map

$$
: \mu \rightarrow \int_{K_{R}} P(t, \sigma) \mathrm{d} \mu(t,[[\sigma]], v)
$$


is continuous. Since we have endowed $B_{R}$ with the weak topology, the map Hom: $K_{R} \rightarrow \mathbf{R}$ is continuous; as a consequence, the map

$$
: \mu \rightarrow \int_{K_{R}} \operatorname{Hom}(v) \mathrm{d} \mu(t,[[\sigma]], v)
$$

is continuous too. Let us prove that the map

$$
: \mu \rightarrow \int_{K_{R}} \frac{1}{2}\|v\|^{2} \mathrm{~d} \mu(t,[[\sigma]], v)
$$

is l. s. c.. To do this, we let $\left\{\psi_{n}\right\}_{n \geq 1}$ be a sequence dense in $B_{R}$ for the strong topology of $L^{2}(I)$, and we define

$$
\begin{gathered}
g_{n}: L^{2}(I) \rightarrow \mathbf{R} \\
g_{n}(v)=\sup \left\{\left\langle v, \psi_{i}\right\rangle-\frac{1}{2}\left\|\psi_{i}\right\|^{2}: i \in(1, \ldots, n)\right\} .
\end{gathered}
$$

It is a standard fact that, if $\|v\| \leq R$, then

$$
\frac{1}{2}\|v\|^{2}=\sup _{\|\psi\| \leq R}\left\{\langle v, \psi\rangle-\frac{1}{2}\|\psi\|^{2}\right\} .
$$

Since $\left\{\psi_{i}\right\}$ is dense in $B_{R}$, the last formula implies that, if $v \in B_{R}$, then

$$
g_{n}(v) \nearrow \frac{1}{2}\|v\|^{2} \quad \forall v \in B_{R}
$$

Formula (3.2) and Cauchy-Schwarz imply the first inequality below; since $v$ and $\psi_{1}$ are in $B_{R}$, also the second one follows.

$$
g_{n}(v) \geq-\|v\| \cdot\left\|\psi_{1}\right\|-\frac{1}{2}\left\|\psi_{1}\right\|^{2} \geq-\frac{3}{2} R^{2} \quad \forall v \in B_{R}
$$

Since $-\frac{3 R^{2}}{2} \in L^{1}\left(K_{R}, \mu\right)$, we can apply monotone convergence and get that

$$
\int_{K_{R}} \frac{1}{2}\|v\|^{2} \mathrm{~d} \mu(t,[[\sigma]], v)=\sup _{n \geq 1} \int_{K_{R}} g_{n}(v) \mathrm{d} \mu(t,[[\sigma]], v) .
$$

Thus, the lower semicontinuity of (3.1) follows, if we prove that each map

$$
: \mu \rightarrow \int_{K_{R}} g_{n}(v) \mathrm{d} \mu(t,[[\sigma]], v)
$$

is continuous. By the definition of the weak* topology, it suffices to prove that each function $g_{n}: K_{R} \rightarrow \mathbf{R}$ is continuous. But this is true because, by (3.2), $g_{n}$ is the sup of a finite family of maps, each of which is continuous on $K_{R}$.

The next corollary follows at once from the last two lemmas.

Corollary 3.3. If $c \in \mathbf{R}$ and $R>0$ is so large that $K_{R}$ is not empty, then there is $\bar{\mu} \in \mathcal{M}_{1}^{R}$ such that

$$
I_{c}(\bar{\mu})=\inf _{\mu \in \mathcal{M}_{1}^{R}} I_{c}(\mu) .
$$

We call $c$-minimal the measures which satisfy the formula above. We want to prove, following [12] and [7], that

1. - for $R$ large, the set of the $c$-minimal measures does not depend on $R$; 
2. - the orbits in the support of a $c$-minimal measure are $c$-minimal.

We need a lemma.

Lemma 3.4. There is a function $R: \mathbf{R} \rightarrow(0,+\infty)$, bounded on bounded sets, such that, for any c-minimal $\sigma \in A C_{\text {mon }}([0,1])$, we have that

$$
\sup _{t \in[0,1]}\left\|\dot{\sigma}_{t}\right\| \leq R(c) \text {. }
$$

Proof. Since $\sigma_{0}, \sigma_{1} \in$ Mon, we have $\sigma_{0}(1-) \leq \sigma_{0}(0)+1$ and $\sigma_{1}(1-) \leq \sigma_{1}(0)+1$; thus, we can find $z_{0}, z_{1} \in \mathbf{Z}$ such that $\sigma_{0}+z_{0}$ and $\sigma_{1}+z_{1}$ have range in $[-1,1]$. We set $\bar{A}=\sigma_{0}+z_{0}, \bar{B}=\sigma_{1}+z_{1}$ and

$$
\tilde{\sigma}_{t}=(1-t) \bar{A}+t \bar{B} \text {. }
$$

We denote, as usual, by $\|\cdot\|_{C^{0}}$ the sup norm; an easy calculation shows that

$$
C_{1}:=\|V\|_{C^{0}\left(S^{1} \times S^{1}\right)}+\|W\|_{C^{0}\left(S^{1}\right)} \geq\|\mathcal{V}\|_{C^{0}\left(S^{1} \times L^{2}\right)}+\|\mathcal{W}\|_{C^{0}\left(L^{2}\right)} .
$$

This implies the first inequality below.

$$
\begin{aligned}
\int_{0}^{1} \mathcal{L}_{c}\left(t, \tilde{\sigma}_{t}, \dot{\tilde{\sigma}}_{t}\right) \mathrm{d} t & =\int_{0}^{1}\left[\frac{1}{2}\|\bar{B}-\bar{A}\|^{2}-\langle c, \bar{B}-\bar{A}\rangle-\mathcal{V}\left(t, \tilde{\sigma}_{t}\right)-\mathcal{W}\left(\tilde{\sigma}_{t}\right)\right] \mathrm{d} t \\
& \leq \frac{1}{2}\|\bar{B}-\bar{A}\|^{2}-\langle c, \bar{B}-\bar{A}\rangle+C_{1} \leq \frac{4}{2}+2|c|+C_{1}=C_{2} .
\end{aligned}
$$

The last inequality above follows from the fact that $\bar{A}$ and $\bar{B}$ have range in $[-1,1]$. Since $\sigma$ is $c$-minimal, and $\sigma_{i}-\tilde{\sigma}_{i} \in L_{\mathbf{Z}}^{2}$ for $i=0,1$, we get that

$$
\int_{0}^{1} \mathcal{L}_{c}\left(t, \sigma_{t}, \dot{\sigma}_{t}\right) \mathrm{d} t \leq \int_{0}^{1} \mathcal{L}_{c}\left(t, \tilde{\sigma}_{t}, \dot{\tilde{\sigma}}_{t}\right) \mathrm{d} t \leq C_{2} .
$$

The first inequality below follows from Cauchy-Schwarz; the second one from the fact that $\frac{1}{4} x^{2}-c^{2} \leq \frac{1}{2} x^{2}-c x$; the third one, from (3.3) and the last one from the formula above.

$$
\begin{aligned}
\frac{1}{4}\left(\int_{0}^{1}\left\|\dot{\sigma}_{t}\right\| \mathrm{d} t\right)^{2}-c^{2} & \leq \frac{1}{4} \int_{0}^{1}\left\|\dot{\sigma}_{t}\right\|^{2} \mathrm{~d} t-c^{2} \leq \int_{0}^{1}\left[\frac{1}{2}\left\|\dot{\sigma}_{t}\right\|^{2}-\left\langle c, \dot{\sigma}_{t}\right\rangle\right] \mathrm{d} t \\
& \leq \int_{0}^{1} \mathcal{L}_{c}\left(t, \sigma_{t}, \dot{\sigma}_{t}\right) \mathrm{d} t+C_{1} \leq C_{2}+C_{1}
\end{aligned}
$$

From this it follows that

$$
\int_{0}^{1}\left\|\dot{\sigma}_{t}\right\| \mathrm{d} t \leq C_{4}
$$

We get as in (3.3) that

$$
\left\|\mathcal{V}^{\prime}\right\|_{C^{0}\left(S^{1} \times L^{2}\right)}+\left\|\mathcal{W}^{\prime}\right\|_{C^{0}\left(L^{2}\right)} \leq\left\|V^{\prime}\right\|_{C^{0}\left(S^{1} \times S^{1}\right)}+\left\|W^{\prime}\right\|_{C^{0}\left(S^{1}\right)} .
$$

Now $\sigma$, being minimal, satisfies $(O D E)_{L a g}$; by the last formula, this implies that

$$
\left\|\ddot{\sigma}_{t}\right\| \leq C_{5} \quad \forall t \in[0,1] .
$$

The last formula and (3.4) imply the thesis. 
Lemma 3.5. Let $R(c)>0$ be as in Lemma 3.4 and let $R \geq R(c)$. Let $\mu$ minimize $I_{c}$ in $\mathcal{M}_{1}^{R}$. Then, the following three points hold.

1) $\mu$ a.e. $(t,[[M]], v) \in K_{R}$ is the initial condition of a c-minimal orbit. Moreover, $\mu$ induces, in a natural way, a measure $\gamma_{0}$ on $K_{R} \cap\{t=0\}$ such that the following happens. If $U$ is c-dominated, for $\gamma_{0}$ a.e. $(0,[[M]], v)$, we have that the curve $\sigma_{s}=\pi_{m o n} \circ \psi_{s}(0, M, v)$ is calibrating for $U$.

2) $\mu$ is supported in $K_{R(c)}$.

3) For the function $\alpha$ defined in Sect. 2, we have that

$$
-\alpha(c)=I_{c}(\mu)=\min _{\nu \in \mathcal{M}_{1}^{R}} I_{c}(\nu)
$$

Proof. We begin to note that point 2) follows from point 1) and Lemma 3.4; before proving point 1), we sketch the proof of 3) given in [7].

We have to prove only the first equality, since the fact that that $\mu$ is minimal in $\mathcal{M}_{1}^{R}$ is one of the hypotheses. Let us consider the projections

$$
\begin{aligned}
& \pi_{\text {mon }}: S^{1} \times \text { Mon }_{\mathbf{Z}} \times L^{2}(I) \rightarrow \text { Mon }_{\mathbf{Z}}, \quad \pi_{L^{2}}: S^{1} \times \operatorname{Mon}_{\mathbf{Z}} \times L^{2}(I) \rightarrow L^{2}(I), \\
& \pi_{m o n \times L^{2}}: S^{1} \times \operatorname{Mon}_{\mathbf{Z}} \times L^{2}(I) \rightarrow \text { Mon }_{\mathbf{Z}} \times L^{2}(I), \\
& \pi_{\text {time }}: S^{1} \times \operatorname{Mon}_{\mathbf{Z}} \times L^{2}(I) \rightarrow S^{1} .
\end{aligned}
$$

Let us consider $\left(\pi_{\text {time }}\right)_{\sharp} \mu$, the marginal of $\mu$ on $S^{1}$; since $\mu$ is invariant by $\psi_{s}$, it is easy to see that $\left(\pi_{\text {time }}\right)_{\sharp} \mu$ is translation-invariant, and thus it must coincide with the Lebesgue measure on $S^{1}$. As a consequence, we can disintegrate $\mu$ as $\mu=\mathcal{L}^{1} \otimes \gamma_{t}$, where $\mathcal{L}^{1}$ is the Lebesgue measure on $S^{1}$ and $\gamma_{t}$ is a probability measure on $\operatorname{Mon}_{\mathbf{Z}} \times B(0, R)$. Using again the fact that $\mu$ is invariant by the flow $\psi_{s}$, we easily see that $\gamma_{t}=\left(\pi_{\operatorname{mon} \times L^{2}} \circ \psi_{t}(0, \cdot, \cdot)\right)_{\sharp} \gamma_{0}$; as a consequence, $\gamma_{0}$ is invariant by the time-one map $\Psi:(M, v) \rightarrow \pi_{m o n \times L^{2}} \circ \psi_{1}(0, M, v)$.

Let now $U: L^{2}(I) \rightarrow \mathbf{R}$ be a fixed point of $T_{1}^{-}$; we have seen in Proposition 2.2 that such a function exists. By Lemma 2.3, $U$ is $c$-dominated, and thus, for $k \in \mathbf{N}$, we have

$$
U \circ \pi_{m o n} \circ \Psi^{k}(M, v)-U \circ \pi_{m o n}(M, v) \leq \int_{0}^{k}\left[\mathcal{L}_{c}\left(t, \sigma_{t}, \dot{\sigma}_{t}\right)+\alpha(c)\right] \mathrm{d} t
$$

for every $\sigma \in A C_{m o n}([0, k])$ with $\sigma_{k}=\pi_{m o n} \circ \Psi^{k}(M, v)$ and $\sigma_{0}=M$. We let $\sigma_{t}^{M, v}=\pi_{m o n} \circ \psi_{t}(0, M, v)$; we consider (3.5) for $k \geq 1$ and $\sigma=\sigma^{M, v}$; we integrate it under $\gamma_{0}$ and we get the inequality below.

$$
\begin{aligned}
0 & =\int_{M o n_{\mathbf{Z}} \times B_{R}}\left[U \circ \pi_{m o n} \circ \Psi^{k}(M, v)-U \circ \pi_{m o n}(M, v)\right] \mathrm{d} \gamma_{0}([[M]], v) \\
& \leq \int_{M o n_{\mathbf{Z}} \times B_{R}} \mathrm{~d} \gamma_{0}([[M]], v) \int_{0}^{k}\left[\mathcal{L}_{c}\left(\psi_{t}(0, M, v)\right)+\alpha(c)\right] \mathrm{d} t \\
& =\int_{0}^{k} \mathrm{~d} t \int_{M o n_{\mathbf{Z}} \times B_{R}}\left[\mathcal{L}_{c}(t, M, v)+\alpha(c)\right] \mathrm{d} \gamma_{t}([[M]], v) \\
& =k \int_{K_{R}}\left[\mathcal{L}_{c}(t, M, v)+\alpha(c)\right] \mathrm{d} \mu(t, M, v) .
\end{aligned}
$$


The first equality above follows because $\gamma_{0}$ is invariant by the time-one map $\Psi$, the second one because $\gamma_{t}=\psi_{t}(0, \cdot, \cdot)_{\sharp} \gamma_{0}$, and the third one because $\mu=$ $\mathcal{L}^{1} \otimes \gamma_{t}$.

Now (3.6) implies that, for $\mu c$-minimal,

$$
I_{c}(\mu) \geq-\alpha(c) \text {. }
$$

We want to prove the opposite inequality. Let $M \in M o n$, we recall from Proposition 2.2 that there is $\sigma \in A C_{m o n}((-\infty, 0])$ with $\sigma_{0}=M$ such that, for any $k \in \mathbf{N}$,

$$
U(M)-U\left(\sigma_{-2 k}\right)=\int_{-2 k}^{0}\left[\mathcal{L}_{c}\left(t, \sigma_{t}, \dot{\sigma}_{t}\right)+\alpha(c)\right] \mathrm{d} t .
$$

Now we use the Krylov-Bogoljubov argument: we consider the map

$$
\Phi_{k}:[-k, k] \rightarrow F_{R}, \quad \Phi_{k}: t \rightarrow\left(t \bmod 1, \sigma_{t-k} \bmod 1, \dot{\sigma}_{t-k}\right)
$$

and the probability measure $\mu_{k}=\left(\Phi_{k}\right)_{\sharp} \nu_{k}$, where $\nu_{k}$ is the Lebesgue measure on $[-k, k]$ normalized to 1 .

Since $\sigma$ is $c$-minimal on $(-\infty, 0]$, Lemma 3.4 implies that $\Phi_{k}([-k, k]) \in$ $F_{R(c)} \subset F_{R}$ for $k \geq 1$.

This implies that $\mu_{k}$ is supported in the compact set $F_{R}$; thus, up to subsequences, $\mu_{k}$ converges weak* to a probability measure $\bar{\mu}$ on $F_{R}$. We assert that $\bar{\mu} \in \mathcal{M}_{1}^{R}$, i.e. that $\bar{\mu}$ is invariant and supported on $K_{R}$. The Kryolov-Bogolyubov construction implies in a standard way that $\bar{\mu}$ is invariant; moreover, $\bar{\mu}$ is supported on the limits of the orbits $\sigma_{t-k}$; but $\sigma_{t-k} \in$ Mon for $t \in(-\infty, k]$, and thus any of its limits $\tilde{\sigma}_{t}$ belongs to Mon for all $t \in \mathbf{R}$.

This and Lemma 3.2 imply the inequality below.

$$
\begin{aligned}
I_{c}(\bar{\mu}) & \leq \liminf _{k \rightarrow+\infty} I_{c}\left(\mu_{k}\right)=\liminf _{k \rightarrow+\infty} \frac{1}{2 k} \int_{-2 k}^{0} \mathcal{L}_{c}\left(t, \sigma_{t}, \dot{\sigma}_{t}\right) \mathrm{d} t \\
& =\liminf _{k \rightarrow+\infty} \frac{1}{2 k}\left[U(M)-U\left(\sigma_{-2 k}\right)-2 k \alpha(c)\right]=-\alpha(c) .
\end{aligned}
$$

The first equality above comes from the definition of $\mu_{k}$, the second one comes from (3.8) and the third one from the fact, which we saw at the beginning of Sect. 2, that $U$ is bounded. Since $\bar{\mu}$ is an invariant probability measure on $K_{R}$, the last formula and (3.7) imply point 3).

By point 3), for $k \in \mathbf{N}$ formula (3.6) collapses to

$$
\begin{aligned}
0 & =\int_{K_{R} \cap\{t=0\}}\left[U \circ \pi_{m o n} \circ \Psi^{k}(M, v)-U \circ \pi_{m o n}(M, v)\right] \mathrm{d} \gamma_{0}([[M]], v) \\
& =\int_{K_{R} \cap\{t=0\}} \mathrm{d} \gamma_{0}([[M]], v) \int_{0}^{k}\left[\mathcal{L}_{c}\left(t, \sigma_{t}^{M, v}, \dot{\sigma}_{t}^{M, v}\right)+\alpha(c)\right] \mathrm{d} t .
\end{aligned}
$$

This and (3.5) imply that, for all $k \in \mathbf{N}$ and $\gamma_{0}$ a.e. ([[M]], $v$ ),

$$
U \circ \pi_{m o n} \circ \Psi^{k}(0, M, v)-U(M)=\int_{0}^{k}\left[\mathcal{L}_{c}\left(t, \sigma_{t}^{M, v}, \dot{\sigma}_{t}^{M, v}\right)+\alpha(c)\right] \mathrm{d} t .
$$

We have seen that, since $U$ is $c$-dominated, this implies that $\sigma^{M, v}$ is $c$-minimal for a.e. ([[M]],v); but this is point 1$)$. 
Now we briefly define, following [12], the two "conjugate mean actions" $\alpha$ and $\beta$.

For starters, we define the rotation number of $\mu \in \mathcal{M}_{1}^{R}$ in the standard way, by duality with the equivariant homology of $L^{2}(I)$. We recall from Proposition 1.1 that, if $S \in C^{1}\left(L^{2}(I)\right)$ and $\mathrm{d} S$ is $L_{\mathbf{Z}}^{2}$ and Group-equivariant, then

$$
\mathrm{d} S=c+\mathrm{d} s
$$

with $c \in \mathbf{R}$; the function $s$, which belongs to $C^{1}\left(L^{2}(I)\right)$, is $L_{\mathbf{Z}}^{2}$ and is Groupequivariant. Let $\mu \in \mathcal{M}_{1}^{R}$; as in [12], the ergodic theorem implies the first equality below.

$$
\begin{aligned}
& \int_{K_{R}}\left\langle\mathrm{~d}_{M} s, v\right\rangle \mathrm{d} \mu(t,[[M]], v) \\
& \quad=\int_{K_{R}} \mathrm{~d} \mu(t,[[M]], v) \lim _{n \rightarrow+\infty} \frac{1}{n} \int_{0}^{n} \frac{\mathrm{d}}{\mathrm{d} \tau} s\left(\pi_{m o n} \circ \psi_{\tau}(t, M, v)\right) \mathrm{d} \tau \\
& \quad=\int_{K_{R}} \lim _{n \rightarrow+\infty} \frac{1}{n}\left[s \circ \pi_{m o n} \circ \psi_{n}(t, M, v)-s \circ \pi_{m o n}(t, M, v)\right] \mathrm{d} \mu(t,[[M]], v)=0 .
\end{aligned}
$$

The last equality above comes from the fact that any $s \in C_{\text {Group }}(\mathbf{T})$ is bounded; we saw this right at the beginning of Sect. 2. As a consequence,

$$
\int_{K_{R}}\left\langle c+\mathrm{d}_{M} s, v\right\rangle \mathrm{d} \mu(t,[[M]], v)
$$

depends only on $c \in \mathbf{R}$. If we define $\rho(\mu)$ as

$$
\rho(\mu)=\int_{K_{R}}\langle 1, v\rangle \mathrm{d} \mu(t,[[M]], v),
$$

we see by the formula above that

$$
\int_{K_{R}}\left\langle c+\mathrm{d}_{M} s, v\right\rangle \mathrm{d} \mu(t,[[M]], v)=c \cdot \rho(\mu)
$$

for all $c \in \mathbf{R}$ and $s \in C^{1}\left(L^{2}(I)\right), L_{\mathbf{Z}}^{2}$ and Group-equivariant. One can look on $\rho(\mu)$ as on the "mean number of turns of all the particles around $S^{1 "}$; indeed, by the ergodic theorem, (3.10) implies the first equality below.

$$
\begin{aligned}
\rho(\mu) & =\int_{K_{R}} \mathrm{~d} \mu(t,[[M]], v) \lim _{n \rightarrow+\infty} \frac{1}{n} \int_{0}^{n} \frac{\mathrm{d}}{\mathrm{d} \tau}\left\langle 1, \pi_{m o n} \circ \psi_{\tau}(t, M, v)\right\rangle \mathrm{d} \tau \\
& =\int_{K_{R}} \mathrm{~d} \mu(t,[[M]], v) \lim _{n \rightarrow+\infty} \frac{1}{n}\left\langle 1,\left[\pi_{m o n} \circ \psi_{n}(t, M, v)-M\right]\right\rangle .
\end{aligned}
$$

Now, since $: x \rightarrow \pi_{m o n} \circ \psi_{n}(t, M, v)(x)$ belongs to $M o n$, it is easy to see that

$$
\lim _{n \rightarrow+\infty} \frac{1}{n}\left[\pi_{m o n} \circ \psi_{n}(t, M, v)(x)-M(x)\right]
$$

does not depend on $x \in I$; actually, it is equal to

$$
\lim _{n \rightarrow+\infty} \frac{1}{n}\left\langle 1,\left[\pi_{m o n} \circ \psi_{n}(t, M, v)-M\right]\right\rangle,
$$

yielding that 


$$
\rho(\mu)=\lim _{n \rightarrow+\infty} \frac{1}{n}\left[\pi_{m o n} \circ \psi_{n}(t, M, v)(x)-M(x)\right]
$$

for all $x \in I$.

Let the space $\mathcal{C}_{1}$ be as in the end of Sect. 1; it is a standard fact (see [12]) that $S^{1} \times \mathcal{C}_{1} \times \mathcal{C}_{1}$ (the phase space of a single particle), which is invariant by the Euler-Lagrange flow of $\mathcal{L}$, contains measures of any rotation number $\rho \in \mathbf{R}$; as a consequence, if $\rho \in \mathbf{R}$ is given and $R>0$ is large enough, $\mathcal{M}_{1}^{R}$ contains measures of rotation number $\rho$.

We define

$$
\beta^{R}(\rho)=\min \left\{\int_{K_{R}} \mathcal{L}_{0}(t, M, v) \mathrm{d} \mu(t,[[M]], v): \mu \in \mathcal{M}_{1}^{R} \quad \text { and } \quad \rho(\mu)=\rho\right\}
$$

and

$$
-\alpha^{R}(c)=\min \left\{\int_{K_{R}} \mathcal{L}_{c}(t, M, v) \mathrm{d} \mu(t,[[M]], v): \mu \in \mathcal{M}_{1}^{R}\right\} .
$$

The second minimum is attained by corollary 3.3 ; by Lemma 3.2 , to prove that the first minimum is attained, it suffices to prove that the set

$$
\left\{\mu \in \mathcal{M}_{1}^{R}: \rho(\mu)=\rho\right\}
$$

is compact. Since $\mathcal{M}_{1}^{R}$ is compact, it suffices to prove that $: \mu \rightarrow \rho(\mu)$ is continuous for the weak* topology on $\mathcal{M}_{1}^{R}$; this in turn follows from the fact that the integral on the right hand side of (3.10) is a continuous function of $\mu$; we saw this in the proof of Lemma 3.2, where we called it $\operatorname{Hom}(v)$.

By point 3) of Lemma 3.5, we get that, for $R \geq R(c), \alpha^{R}(c)=\alpha(c)$. By point 2) of the same lemma, the $c$-minimal measures are supported in $K_{R(c)}$. By definition, $\beta^{R}$ is decreasing in $R$; we set

$$
\beta(\rho)=\inf _{R>0} \beta^{R}(\rho)=\lim _{R \rightarrow+\infty} \beta^{R}(\rho) .
$$

It is easy to see that $\alpha$ and $\beta$ are convex; we recall the proof, which is identical to [12], that each of them is the Legendre transform of the other one. Indeed,

$$
\begin{aligned}
\beta^{*}(c) & =\sup _{\rho}\{\rho \cdot c-\beta(\rho)\}=\sup _{\rho, R}\left\{\rho \cdot c-\beta_{R}(\rho)\right\} \\
& =\sup _{\rho, R} \sup \left\{\rho \cdot c-\int_{K_{R}} \mathcal{L} \mathrm{d} \mu: \rho(\mu)=\rho, \quad \mu \in \mathcal{M}_{1}^{R}\right\} \\
& =\sup _{R} \sup _{\mu \in \mathcal{M}_{1}^{R}}\left(-\int_{K_{R}} \mathcal{L}_{c} \mathrm{~d} \mu\right)=\alpha(c)
\end{aligned}
$$

where the last but one equality comes from (3.10) and the last one the fact that $\alpha^{R}(c)=\alpha(c)$ for $R$ large enough. The proof that $\beta$ is the Legendre transform of $\alpha$ is analogous.

The fact that $\alpha$ and $\beta$ are each the Legendre transform of the other, implies that both have superlinear growth. Since $\alpha$ is the Legendre transform of $\beta$, we have that $\beta(\rho)=c \cdot \rho-\alpha(c)$ for any $c \in \partial \beta(\rho)$; as a consequence, 
$\beta(\rho)$ is attained exactly on the $c$-minimal measures, for $c \in \partial \beta(\rho)$; since $\beta$ is superlinear, $\partial \beta(\rho)$ is compact, and thus

$$
R_{\rho}:=\sup _{c \in \partial \beta(\rho)} R(c)
$$

is finite. In other words, for $R \geq R_{\rho}$, the set of measures $\mu$ on $K_{R}$ such that $\rho(\mu)=\rho$ and $I_{c}(\mu)=\beta^{R}(\rho)$ does not depend on $R$; or $\beta^{R}(\rho)=\beta(\rho)$ for $R$ large enough.

We define $\hat{M a t} t_{c}$ as the closure of the union of the supports of all the $c$-minimal measures; we define

$$
\begin{aligned}
\tilde{M a} t_{c}:= & (\Pi, i d)^{-1}\left\{\hat{M a t} t_{c} \cap\{t=0\}\right\} \subset \text { Mon } \times L^{2}(I), \\
& \text { Mat }_{c}:=\pi_{\text {mon }}\left(\tilde{M} a t_{c}\right) \subset \text { Mon }
\end{aligned}
$$

where the projection $\Pi$ was defined at the beginning of this section. In other words, $\tilde{M a} t_{c}$ is the set of all initial conditions $(M, v)$ in $M o n \times L^{2}(I)$ such that $(\Pi, i d) \circ \psi_{s}(0, M, v)$ lies in the support of a $c$-minimal measure; Mat $_{c}$ is what we get from this set forgetting the velocity variable.

\section{The Aubry set}

In this section, we define the Aubry set in terms of the operators $T_{1}^{-}$and $T_{-1}^{+}$; we shall check that the arguments of [7] continue to work.

Lemma 4.1. If $U$ is c-dominated, if $M \in M a t_{c}$ and $n \in \mathbf{N}$, then

$$
\left(T_{n}^{-} U\right)(M)=\left(T_{-n}^{+} U\right)(M)=U(M) \text {. }
$$

Proof. Since $U$ is $c$-dominated, $U(M) \geq\left(T_{-n}^{+} U\right)(M)$ by point 1) of Lemma 2.3. Since $M \in M a t_{c}$, we have that formula (3.9) holds; now (3.9) immediately implies that $\left(T_{-n}^{+} U\right)(M) \geq U(M)$, and we are done.

Theorem 4.2. If $U \in C_{\text {Group }}(\mathbf{T})$ is c-dominated, there are a fixed point $U^{-}$of $T_{1}^{-}$and a fixed point $U^{+}$of $T_{-1}^{+}$which satisfy the following points.

1) $U(M)=U^{-}(M)=U^{+}(M)$ if $M \in M a t_{c}$.

2) $U^{+}(M) \leq U(M) \leq U^{-}(M)$ for all $M \in L^{2}(I)$.

3) $U^{-}$is the smallest of the fixed points of $T_{1}^{-}$which are larger than $U$, and $U^{+}$is the largest of the fixed points of $T_{-1}^{+}$which are smaller than $U$. In other words,

(a) - if $U_{1}^{-}$is a fixed point of $T_{1}^{-}$such that $U \leq U_{1}^{-}$, then $U^{-} \leq U_{1}^{-}$, and

(b) - if $U_{1}^{+}$is a fixed point of $T_{-1}^{+}$such that $U \geq U_{1}^{+}$, then $U^{+} \geq U_{1}^{+}$.

4) The sequences $T_{n}^{-} U$ and $T_{-n}^{+} U$ converge to $U^{-}$and $U^{+}$respectively, uniformly on $L^{2}(I)$.

5) If $U^{-}$is a fixed point of $T_{1}^{-}$, then there is a fixed point $U^{+}$of $T_{-1}^{+}$such that $U^{-}=U^{+}$on Mat ; moreover, $U^{+} \leq U^{-}$on Mon.

Proof. We only sketch the proof, since it is identical to [7]. 
We note that

$$
T_{n+1}^{-} U=T_{n}^{-} \circ T_{1}^{-} U \geq T_{n}^{-} U
$$

where the equality comes from the semigroup property, and the inequality from point 1) of Lemma 2.3 and the fact, which we saw at the beginning of Sect. 2, that $T_{n}^{-}$is monotone. Thus, $T_{n}^{-} U$ is an increasing sequence. Moreover, by point 2) of Proposition 2.1, $T_{n}^{-} U$ is $L$-Lipschitz for dist $\mathbf{s}$, for some $L>0$ independent on $n$. Thus, $T_{n}^{-} U$ quotients on the compact set $\mathbf{S}$ as an increasing sequence of $L$-Lipschitz functions. By Lemma 4.1, $T_{n}^{-} U=U$ on Mat $t_{c}$; since $\mathbf{S}$ is compact, and $T_{n}^{-} U$ is uniformly Lipschitz, the sequence $T_{n}^{-} U$ is bounded in the sup norm. Thus, $T_{n}^{-} U$ quotients to an increasing, bounded, uniformly Lipschitz sequence of functions on $\mathbf{S}$; as a result, $T_{n}^{-} U$ converges uniformly to a $L$-Lipschitz function $U^{-}$on $\mathbf{S}$. We go back to $L^{2}(I)$; what we just said implies that $T_{n}^{-} U$ converges uniformly to $U^{-}$in $L^{2}(I)$; since $\operatorname{dist}_{\mathbf{S}}([u],[v])=\operatorname{dist}_{\text {weak }}(u, v) \leq\|u-v\|$, we get that $U^{-}$is $L$-Lipschitz on $L^{2}(I)$. Since $T_{n}^{-} U \geq U$, we get that $U^{-} \geq U$. Since $T_{n}^{-} U=U$ on $M a t_{c}$, we get that $U^{-}=U$ on Mat $_{c}$. Thus, $U^{-}$(and $U^{+}$, with the same proof) satisfies points 1), 2) and 4).

We saw right after the definition of $\Lambda_{c, l}$ that the map : $U \rightarrow T_{1}^{-} U$ is continuous for the sup norm; this implies the second equality below, while the first and last one follow by point 4).

$$
T_{1}^{-} U^{-}=T_{1}^{-}\left(\lim _{n \rightarrow+\infty} T_{n}^{-} U\right)=\lim _{n \rightarrow+\infty} T_{(n+1)}^{-} U=U^{-} .
$$

This proves that $U^{-}$is a fixed point of $T_{1}^{-}$.

We prove 3 ); let $U_{1}^{-}$be as in this point. The first equality below is point 4); the inequality is the fact, which we saw before Proposition 2.2 , that $T_{n}^{-}$is monotone: $T_{n}^{-}\left(V_{1}\right) \leq T_{n}^{-}\left(V_{2}\right)$ if $V_{1} \leq V_{2}$.

$$
U^{-}(M)=\lim _{n \rightarrow+\infty} T_{n}^{-} U(M) \leq \lim _{n \rightarrow+\infty} T_{n}^{-} U_{1}^{-}(M)=U_{1}^{-}(M) .
$$

The last equality above follows because $U_{1}^{-}$is a fixed point of $T_{1}^{-}$.

We prove 5). Let $U^{-}$be a fixed point of $T_{-1}^{-}$; by point 4 ), we can build $U^{+}$as the limit of $T_{-n}^{+}\left(U^{-}\right)$as $n \rightarrow+\infty$; by point 1$), U^{-}=U^{+}$on $M a t_{c}$; applying point 2) with $U=U^{-}$, we get that $U^{+} \leq U^{-}$.

Lemma 4.3. Let $\mathcal{U}$ be an open neighbourhood of $\hat{M a t} t_{c}$. Then, there is $t(\mathcal{U})>0$ with the following property. If $t \geq t(\mathcal{U})$ and $\gamma \in A C_{\text {mon }}([0, t])$ is c-minimal, then there is $s \in[0, t] \cap \mathbf{N}$ with $\left(s, \gamma_{s}, \dot{\gamma}_{s}\right) \in \mathcal{U}$.

Proof. The proof of this lemma is identical to [7]; essentially, it follows from the fact that, as $k \rightarrow+\infty$, the push-forward of the normalized Lebesgue measure on $[0, k]$ by the map $: s \rightarrow\left(s, \gamma_{s}, \dot{\gamma}_{s}\right)$ accumulates on a $c$-minimal measure. We used this fact in proving point 3) of Lemma 3.5.

Proposition 4.4. Let $U \in C_{\mathrm{Group}}(\mathbf{T})$ be c-dominated. Then, there is a unique couple $\left(U^{-}, U^{+}\right)$such that $U^{-}$is a fixed point of $T_{1}^{-}, U^{+}$is a fixed point of $T_{-1}^{+}$and $U^{-}=U^{+}=U$ on Mat . Moreover, $U^{+} \leq U^{-}$. 
Proof. Existence of the couple $\left(U^{-}, U^{+}\right)$follows from Theorem 4.2. We prove uniqueness. Let $\left(\tilde{U}^{-}, \tilde{U}^{+}\right)$be another such couple and let $M \in$ Mon; since $\tilde{U}^{-}$is a fixed point of $T_{1}^{-}$, by point 2) of Proposition 2.2 there is $\sigma \in A C_{l o c}((-\infty, 0])$ such that $\sigma_{0}=M$ and, for all $k \in \mathbf{N}$,

$$
\tilde{U}^{-}(M)-\tilde{U}^{-}\left(\sigma_{-k}\right)=\int_{-k}^{0}\left[\mathcal{L}_{c}\left(t, \sigma_{t}, \dot{\sigma}_{t}\right)+\alpha(c)\right] \mathrm{d} t .
$$

By Lemma 4.3, there is a sequence $k_{j} \rightarrow+\infty$ such that $\sigma_{-k_{j}} \rightarrow N \in$ Mat $_{c}$. Since $\tilde{U}^{-}$is continuous, the formula above implies that, in the formula below, the limit on the right exists and it is equal to the expression on the left.

$$
\tilde{U}^{-}(M)-\tilde{U}^{-}(N)=\lim _{j \rightarrow+\infty} \int_{-k_{j}}^{0}\left[\mathcal{L}_{c}\left(t, \sigma_{t}, \dot{\sigma}_{t}\right)+\alpha(c)\right] \mathrm{d} t .
$$

Using the fact that $U^{-}$is $c$-dominated, we get that

$$
U^{-}(M)-U^{-}(N) \leq \lim _{j \rightarrow+\infty} \int_{-k_{j}}^{0}\left[\mathcal{L}_{c}\left(t, \sigma_{t}, \dot{\sigma}_{t}\right)+\alpha(c)\right] \mathrm{d} t .
$$

Since $N \in M a t_{c}$, we have that $U^{-}(N)=U(N)=\tilde{U}^{-}(N)$; from this and the last two formulas we get that

$$
U^{-}(M) \leq \tilde{U}^{-}(M) \quad \forall M \in M o n
$$

which implies in the usual way that

$$
U^{-}(M) \leq \tilde{U}^{-}(M) \quad \forall M \in L^{2}(I) .
$$

Exchanging the rôles of $\tilde{U}^{-}$and $U^{-}$, we get the opposite inequality; this proves the first assertion of the lemma.

The last assertion, i.e. that $U^{+} \leq U^{-}$, follows, in the obvious way, from uniqueness and point 2) of Theorem 4.2.

Definition. A pair of functions $U^{-}, U^{+} \in C_{\text {Group }}(\mathbf{T})$ is said to be conjugate if $U^{-}$is a fixed point of $T_{1}^{-}, U^{+}$is a fixed point of $T_{-1}^{+}$and $U^{+}=U^{-}$on Mat $_{c}$. We denote by $\mathcal{D}$ the set of the couples $\left(U^{-}, U^{+}\right)$of conjugate functions. By Proposition 2.2, there is a $c$-dominated function $U$; thus, by Proposition 4.4, $\mathcal{D}$ is not empty.

Always by Proposition 4.4, if $\left(U^{-}, U^{+}\right) \in \mathcal{D}$, then $U^{+} \leq U^{-}$.

We forego the easy proof that $\mathcal{D}$ is closed in $C\left(L^{2}(I), \mathbf{R}\right) \times C\left(L^{2}(I), \mathbf{R}\right)$.

Definition. For $\left(U^{-}, U^{+}\right) \in \mathcal{D}$, we set

$$
\mathcal{I}_{\left(U^{-}, U^{+}\right)}=\left\{M \in \text { Mon : } U^{-}(M)=U^{+}(M)\right\} .
$$

Let $\left(U^{-}, U^{+}\right) \in \mathcal{D}$; then, by definition of conjugate couple,

$$
\mathrm{Mat}_{c} \subset \mathcal{I}_{\left(U^{-}, U^{+}\right)} .
$$

We note that $\Pi\left(\mathcal{I}_{\left(U^{-}, U^{+}\right)}\right)$is a compact set of $\mathrm{Mon}_{\mathbf{Z}}$; indeed, we have already seen that $M o_{\mathbf{Z}}$ is compact; since the functions $U^{ \pm}$are continuous, $\mathcal{I}_{\left(U^{-}, U^{+}\right)}$ is a closed set of Mon, implying that $\Pi\left(\mathcal{I}_{\left(U^{-}, U^{+}\right)}\right)$is a closed set of $M o n_{\mathbf{Z}}$. 
Theorem 4.5. Let $\left(U^{-}, U^{+}\right) \in \mathcal{D}$ and let $M \in \mathcal{I}_{\left(U^{-}, U^{+}\right)}$. Then, there is a unique c-minimal curve $\gamma \in A C_{\text {mon }}(\mathbf{R})$ such that $\gamma_{0}=M$ and, for all $m \leq n \in \mathbf{Z}$,

$$
U^{ \pm}\left(\gamma_{n}\right)-U^{ \pm}\left(\gamma_{m}\right)=\int_{m}^{n}\left[\mathcal{L}_{c}\left(t, \gamma_{t}, \dot{\gamma}_{t}\right)+\alpha(c)\right] d t .
$$

In other words, $\gamma$ is calibrating both for $U^{-}$and for $U^{+}$. Moreover, $U^{ \pm}$ is Fréchet differentiable at $M$ and

$$
\mathrm{d}_{M} U^{+}=\mathrm{d}_{M} U^{-}=-c+\dot{\gamma}_{0} .
$$

Proof. Let $M \in M o n$; since $U^{-}$and $U^{+}$are fixed points of $T_{-1}^{-}$and $T_{1}^{+}$ respectively, we can apply point 2) of Proposition 2.2 and get that there are two minimal curves, $\gamma^{-} \in A C_{l o c}((-\infty, 0])$ and $\gamma^{+} \in A C_{l o c}([0,+\infty))$, such that

$$
\gamma_{0}^{-}=\gamma_{0}^{+}=M
$$

and, for any $n \in \mathbf{N}$ and $-m \in \mathbf{N}$,

$$
\left\{\begin{array}{l}
U^{-}\left(\gamma_{0}^{-}\right)-U^{-}\left(\gamma_{m}^{-}\right)=\int_{m}^{0}\left[\mathcal{L}_{c}\left(t, \gamma_{t}^{-}, \dot{\gamma}_{t}^{-}\right)+\alpha(c)\right] \mathrm{d} t \\
U^{+}\left(\gamma_{n}^{+}\right)-U^{+}\left(\gamma_{0}^{+}\right)=\int_{0}^{n}\left[\mathcal{L}_{c}\left(t, \gamma_{t}^{+}, \dot{\gamma}_{t}^{+}\right)+\alpha(c)\right] \mathrm{d} t .
\end{array}\right.
$$

We define

$$
\gamma_{t}= \begin{cases}\gamma_{t}^{-} & t \leq 0 \\ \gamma_{t}^{+} & t \geq 0\end{cases}
$$

and we get, by (4.3), that

$$
\int_{m}^{n}\left[\mathcal{L}_{c}\left(t, \gamma_{t}, \dot{\gamma}_{t}\right)+\alpha(c)\right] \mathrm{d} t=U^{+}\left(\gamma_{n}\right)-U^{-}\left(\gamma_{m}\right)+\left[U^{-}\left(\gamma_{0}\right)-U^{+}\left(\gamma_{0}\right)\right] .
$$

We prove that, if $M \in \mathcal{I}_{\left(U^{-}, U^{+}\right)}$, then $\gamma$ satisfies (4.1); clearly, up to integer translations, we can always suppose that $m<0<n$. The first inequality below comes from the fact that $U^{-} \geq U^{+}$; the first equality comes from the fact that $\gamma_{0}=M \in \mathcal{I}_{\left(U^{-}, U^{+}\right)}$; the second one comes from (4.4). The last inequality comes from the fact that $U^{-}$is $c$-dominated.

$$
\begin{aligned}
& U^{-}\left(\gamma_{n}\right)-U^{-}\left(\gamma_{m}\right) \geq U^{+}\left(\gamma_{n}\right)-U^{-}\left(\gamma_{m}\right)=U^{+}\left(\gamma_{n}\right)-U^{-}\left(\gamma_{m}\right)+\left[U^{-}\left(\gamma_{0}\right)-U^{+}\left(\gamma_{0}\right)\right] \\
& =\int_{m}^{n}\left[\mathcal{L}_{c}\left(t, \gamma_{t}, \dot{\gamma}_{t}\right)+\alpha(c)\right] \mathrm{d} t \geq U^{-}\left(\gamma_{n}\right)-U^{-}\left(\gamma_{m}\right) .
\end{aligned}
$$

This formula implies (4.1) for $U^{-}$; the proof for $U^{+}$is analogous.

We saw above that, if $U$ is $c$-dominated and $\gamma$ satisfies (4.1), i.e. it is calibrating, then $\gamma$ is $c$-minimal. This gives existence.

We prove uniqueness. Let $\tilde{\gamma}$ be any curve such that $\tilde{\gamma}_{0}=M$ and such that (4.1) holds. If we define

$$
\hat{\gamma}_{t}= \begin{cases}\gamma_{t} & t \leq 0 \\ \tilde{\gamma}_{t} & t \geq 0\end{cases}
$$

we see as above that $\hat{\gamma}$ satisfies (4.1) and thus it is $c$-minimal; since $c$-minimal curves are $C^{2}$, we get that $\dot{\gamma}_{0}=\dot{\tilde{\gamma}}_{0}$; since both curves satisfy $(O D E)_{\text {Lag }}$, we get that $\tilde{\gamma}=\gamma$.

Formula (4.2) comes from (4.1) and point 5) of Theorem 2.7. 
Definition. Let $\left(U^{-}, U^{+}\right) \in \mathcal{D}$; in view of Theorem 4.5, we can define

$$
\begin{aligned}
\tilde{\mathcal{I}}_{\left(U^{-}, U^{+}\right)} & =\left\{\left(M, c+\mathrm{d}_{M} U^{-}\right): M \in \mathcal{I}_{\left(U^{-}, U^{+}\right)}\right\} \\
& =\left\{\left(M, c+\mathrm{d}_{M} U^{+}\right): M \in \mathcal{I}_{\left(U^{-}, U^{+}\right)}\right\}
\end{aligned}
$$

where the derivatives are in the Fréchet sense.

Theorem 4.6. 1. Let $\left(U^{-}, U^{+}\right) \in \mathcal{D}$. Then, the projection

$$
\pi_{m o n}: \tilde{\mathcal{I}}_{\left(U^{-}, U^{+}\right)} \rightarrow \mathcal{I}_{\left(U^{-}, U^{+}\right)}
$$

is bi-Lipschitz.

2. The set $\tilde{\mathcal{I}}_{\left(U^{-}, U^{+}\right)}$is invariant by the time-one map $\Psi$ of the EulerLagrange flow of $\mathcal{L}$, and it contains the set $\tilde{M}$ at $t_{c}$ defined at the end of Sect. 3. Moreover, $(\Pi \times i d)\left(\tilde{\mathcal{I}}_{\left(U^{-}, U^{+}\right)}\right)$is compact in $M_{\mathbf{Z}} \times L^{2}(I)$; we recall that $\Pi:$ Mon $\rightarrow$ Mon $_{\mathbf{Z}}$ is the projection.

3. If $(M, v) \in \tilde{\mathcal{I}}_{\left(U^{-}, U^{+}\right)}$, and if $\gamma_{t}=\pi_{m o n} \circ \psi_{t}(0, M, v)$, then, for $m \leq n \in \mathbf{Z}$,

$$
U^{ \pm}\left(\gamma_{n}\right)-U^{ \pm}\left(\gamma_{m}\right)=\int_{m}^{n}\left[\mathcal{L}_{c}\left(t, \gamma_{t}, \dot{\gamma}_{t}\right)+\alpha(c)\right] d t .
$$

Proof. Let $(M, v) \in \tilde{\mathcal{I}}_{\left(U^{-}, U^{+}\right)}$and let $\gamma_{t}=\pi_{m o n} \circ \psi_{t}(0, M, v)$; this mean that $\dot{\gamma}_{0}$ satisfies formula (4.2); by the uniqueness part of Theorem 4.5, it satisfies (4.1) too, and this yields point 3 ).

Since $\gamma_{0}=M$, setting $m=-1$ and $n=1$ in point 3 ) of the present theorem, we see that $M \in A_{U^{-}}$; the set $A_{U^{-}}$has been defined before theorem 2.7. Since $M$ is arbitrary in $\mathcal{I}_{\left(U^{-}, U^{+}\right)}$, we get that $\mathcal{I}_{\left(U^{-}, U^{+}\right)} \subset A_{U^{-}}$. Point 1) follows by this and point 5) of theorem 2.7 .

Point 2): the fact that $(\Pi \times i d)\left(\tilde{\mathcal{I}}_{\left(U^{-}, U^{+}\right)}\right.$is compact follows from point 1 ) and the fact that $\Pi\left(\mathcal{I}_{\left(U^{-}, U^{+}\right)}\right)$is compact, which we proved just before theorem 4.5.

We prove that $\tilde{\mathcal{I}}_{\left(U^{-}, U^{+}\right)}$is invariant by $\Psi$. Let $\gamma$ be as in point 3$)$; we have that

$$
\left\{\begin{array}{l}
U^{-}\left(\gamma_{1}\right)-U^{-}\left(\gamma_{-n}\right)=\int_{-n}^{1}\left[\mathcal{L}_{c}\left(t, \gamma_{t}, \dot{\gamma}_{t}\right)+\alpha(c)\right] \mathrm{d} t \\
U^{+}\left(\gamma_{n}\right)-U^{+}\left(\gamma_{1}\right)=\int_{1}^{n}\left[\mathcal{L}_{c}\left(t, \gamma_{t}, \dot{\gamma}_{t}\right)+\alpha(c)\right] \mathrm{d} t
\end{array}\right.
$$

Let us suppose by contradiction that $\gamma_{1} \notin \mathcal{I}_{\left(U^{-}, U^{+}\right)}$, i.e. that $U^{-}\left(\gamma_{1}\right)-$ $U^{+}\left(\gamma_{1}\right)>0$; summing the two formulas above, this implies that

$$
U^{+}\left(\gamma_{n}\right)-U^{-}\left(\gamma_{-n}\right)<\int_{-n}^{n}\left[\mathcal{L}_{c}\left(t, \gamma_{t}, \dot{\gamma}_{t}\right)+\alpha(c)\right] \mathrm{d} t .
$$

On the other side, since $\gamma_{0} \in \mathcal{I}_{\left(U^{-}, U^{+}\right)}$, we have that $U^{-}\left(\gamma_{0}\right)-U^{+}\left(\gamma_{0}\right)=0$; arguing as above, this implies that

$$
U^{+}\left(\gamma_{n}\right)-U^{-}\left(\gamma_{-n}\right)=\int_{-n}^{n}\left[\mathcal{L}_{c}\left(t, \gamma_{t}, \dot{\gamma}_{t}\right)+\alpha(c)\right] \mathrm{d} t .
$$

This contradiction proves that $\gamma_{1} \in \mathcal{I}_{\left(U^{-}, U^{+}\right)}$; since

$$
-c+\dot{\gamma}_{1}=\mathrm{d}_{\gamma_{1}} U^{+}=\mathrm{d}_{\gamma_{1}} U^{-}
$$


by (4.2), we get that that $\tilde{\mathcal{I}}_{\left(U^{-}, U^{+}\right)}$is invariant by $\Psi$.

The fact that $\mathrm{Mat}_{c} \subset \mathcal{I}_{\left(U^{-}, U^{+}\right)}$follows from the definition of conjugate pair; to prove that $\tilde{M} a t_{c} \subset \tilde{\mathcal{I}}_{\left(U^{-}, U^{+}\right)}$, we recall that, in formula (3.9, we have shown that, if $(M, v) \in \tilde{M} a t_{c}$ and $\left(\gamma_{0}, \dot{\gamma}_{0}\right)=(M, v)$, then $\gamma$ satisfies (4.1); by the uniqueness of theorem 4.5 , we get that $\dot{\gamma}_{0}=c+\mathrm{d}_{M} U^{-}\left(\gamma_{0}\right)$, i.e. that $\tilde{M} a t_{c} \subset \tilde{\mathcal{I}}_{\left(U^{-}, U^{+}\right)}$.

Definition. We define the Aubry set $\mathcal{A}_{c}$ and the Mañe set $\mathcal{M} \mathcal{N}_{c}$ in the following way.

$$
\begin{aligned}
& \mathcal{A}_{c}=\bigcap_{\left(U^{-}, U^{+}\right) \in \mathcal{D}} \mathcal{I}_{\left(U^{-}, U^{+}\right)}, \quad \tilde{\mathcal{A}}_{c}=\bigcap_{\left(U^{-}, U^{+}\right) \in \mathcal{D}} \tilde{\mathcal{I}}_{\left(U^{-}, U^{+}\right)} \\
& \mathcal{M} \mathcal{N}_{c}=\bigcup_{\left(U^{-}, U^{+}\right) \in \mathcal{D}} \mathcal{I}_{\left(U^{-}, U^{+}\right)}, \quad \\
& \mathcal{M} N_{c}=\bigcup_{\left(U^{-}, U^{+}\right) \in \mathcal{D}} \tilde{\mathcal{I}}_{\left(U^{-}, U^{+}\right)} .
\end{aligned}
$$

Theorem 4.7. 1) The quotiented Aubry sets $\Pi\left(\mathcal{A}_{c}\right)$ and $(\Pi \times i d)\left(\tilde{\mathcal{A}}_{c}\right)$ are compact; we have that Mat $\subset \subset \mathcal{A}_{c}$ and $\tilde{M} a t_{c} \subset \tilde{\mathcal{A}}_{c}$. Moreover, $\tilde{\mathcal{A}}_{c}$ is invariant by the time-one map $\Psi$.

2) There is a pair $\left(U^{-}, U^{+}\right) \in \mathcal{D}$ such that $\mathcal{A}_{c}=\mathcal{I}_{\left(U^{-}, U^{+}\right)}$.

3) The map $\pi_{\text {mon }}: \tilde{\mathcal{A}}_{c} \rightarrow \mathcal{A}_{c}$ is bi-Lipschitz.

Proof. By definition, each $\Pi\left(\mathcal{I}_{\left(U^{-}, U^{+}\right.}\right)$is compact and contains $\Pi\left(\right.$ Mat $\left._{c}\right)$; moreover, by point 2$)$ of theorem 4.6 , each $(\Pi \times i d)\left(\tilde{\mathcal{I}}_{\left(U^{-}, U^{+}\right)}\right)$is compact, invariant by $\Psi$, and contains $(\Pi \times i d)\left(\tilde{M} a t_{c}\right)$; this implies point 1$)$.

We note that point 2 ) and theorem 4.6 imply point 3 ); actually, point 3 ) is also implied directly by theorem 4.6, because the restriction of a Lipschitz map to a smaller set is Lipschitz.

We prove point 2). First of all, we restrict our conjugate couples to Mon, and quotient them on $M_{\text {on }} \mathbf{Z}$; in other words, we look at them as functions in $C\left(\operatorname{Mon}_{\mathbf{Z}}, \mathbf{R}\right)$. This is justified by the fact, which we saw in Sect. 1, that any $U \in C\left(\operatorname{Mon}_{\mathbf{Z}}, \mathbf{R}\right)$ can be uniquely extended to a function in $C_{\text {Group }}(\mathbf{T})$.

Since $\operatorname{Mon}_{\mathbf{Z}}$ is a compact metric space, $C\left(\operatorname{Mon}_{\mathbf{Z}}, \mathbf{R}\right)$ is separable; since $\mathcal{D}$ is a closed set of $C\left(\operatorname{Mon}_{\mathbf{Z}}, \mathbf{R}\right) \times C\left(\operatorname{Mon}_{\mathbf{Z}}, \mathbf{R}\right)$, we can find a dense sequence $\left\{\left(U_{n}^{+}, U_{n}^{-}\right)\right\}_{n \geq 1} \subset \mathcal{D}$. Since $\left\{U_{n}^{ \pm}\right\}$is a sequence of fixed points of $T_{ \pm 1}^{ \pm}$, it is equilipschitz by Proposition 2.2. We note that, if $\left(U_{n}^{+}, U_{n}^{-}\right) \in \mathcal{D}$ and $a_{n} \in \mathbf{R}$, then $\left(U_{n}^{+}+a_{n}, U_{n}^{-}+a_{n}\right)$ is a conjugate pair too; since $M$ on $_{\mathbf{Z}}$ has finite diameter, since $U_{n}^{ \pm}$is equilipschitz and $U_{n}^{+}=U_{n}^{-}$on $M a t_{c}$, we can choose $a_{n}$ in such a way that $U_{n}^{ \pm}+a_{n}$ is equibounded. Setting $\tilde{U}_{n}^{ \pm}=U_{n}^{ \pm}+a_{n}$, we get that the two series below converge uniformly to two Lipschitz functions on $\mathrm{Mon}_{\mathbf{Z}}$, which we call $\tilde{U}^{-}$and $\tilde{U}^{+}$respectively.

$$
\tilde{U}^{-}=\sum_{n \geq 1} \frac{1}{2^{n}} \tilde{U}_{n}^{-}, \quad \tilde{U}^{+}=\sum_{n \geq 1} \frac{1}{2^{n}} \tilde{U}_{n}^{+} .
$$

Since $\tilde{U}_{n}^{-}=\tilde{U}_{n}^{+}$on $M a t_{c}$, we get that $\tilde{U}^{-}=\tilde{U}^{+}$on $M a t_{c}$. Since $\tilde{U}^{-}$and $\tilde{U}^{+}$are convex combinations of $c$-dominated functions, it follows easily that they are $c$-dominated; by points 1 ) and 2) of theorem 4.2, we can find $U^{-}$, 
a fixed point of $T_{-1}^{-}$, satisfying $U^{-} \geq \tilde{U}^{-}$, and $U^{-}=\tilde{U}^{-}$on $M a t_{c}$. Analogously, there is $U^{+}$, a fixed point of $T_{1}^{+}$, satisfying $U^{+} \leq \tilde{U}^{+}$, with equality on $M a t_{c}$. Since $\tilde{U}^{-}=\tilde{U}^{+}$on $M a t_{c}$, we have that $U^{-}=U^{+}$on $M a t_{c}$, and thus $\left(U^{-}, U^{+}\right) \in \mathcal{D}$. As a consequence,

$$
\mathcal{A}_{c} \subset \mathcal{I}_{\left(U^{-}, U^{+}\right)} .
$$

On the other side, since $\left\{\left(U_{n}^{-}, U_{n}^{+}\right)\right\}_{n \geq 1}$ is dense in $\mathcal{D}$, we see that, if $M \notin \mathcal{A}_{c}$, then

$$
U_{n}^{+}(M)<U_{n}^{-}(M)
$$

for at least one $n$; this implies that $\tilde{U}_{n}^{+}(M)<\tilde{U}_{n}^{-}(M)$ for at least one $n$. On the other side, $\tilde{U}_{n}^{+} \leq \tilde{U}_{n}^{-}$for all $n$, since $\left(\tilde{U}_{n}^{-}, \tilde{U}_{n}^{+}\right)$is a conjugate pair; by (4.5), this implies that

$$
\tilde{U}^{+}(M)<\tilde{U}^{-}(M) .
$$

We saw above that $U^{+} \leq \tilde{U}^{+}$and $\tilde{U}^{-} \leq U^{-}$; thus, if $M \notin \mathcal{A}_{c}$,

$$
U^{+}(M)<U^{-}(M) \text {. }
$$

Together with (4.6), this implies point 2).

Definition. Given $[[M]],[[N]] \in \operatorname{Mon}_{\mathbf{Z}}$ and $n \in \mathbf{N}$, we define as in $[13]$

$h_{n}([[M]],[[N]])=\min \left\{\int_{0}^{n}\left[\mathcal{L}_{c}\left(t, \gamma_{t}, \dot{\gamma}_{t}\right)+\alpha(c)\right] \mathrm{d} t: \sigma_{0} \in[[M]], \quad \sigma_{n} \in[[N]],\right\}$ and

$$
h_{\infty}([[M]],[[N]])=\liminf _{n \rightarrow+\infty} h_{n}([[M]],[[N]]) .
$$

The minimum in the definition of $h_{n}$ is attained by an argument similar to that of point 4) of Proposition 2.1. Naturally, we have to prove that $h_{\infty}$ is finite; for this, we refer the reader to [13], since the proof is identical.

Lemma 4.8. If $\left(U^{-}, U^{+}\right) \in \mathcal{D}$, then

$$
\forall M_{-}, M_{+} \in \text { Mon, } \quad U^{-}\left(M_{-}\right)-U^{+}\left(M_{+}\right) \leq h_{\infty}\left(\left[\left[M_{-}\right]\right],\left[\left[M_{+}\right]\right]\right) .
$$

Proof. We recall the proof of [7]. By the definition of $h_{\infty}$, we can find a sequence of integers $n_{k} \rightarrow+\infty$ and a minimal $\gamma_{k} \in A C_{m o n}\left(\left[0, n_{k}\right]\right)$ such that

$$
\left\{\begin{array}{l}
h_{\infty}\left(\left[\left[M_{-}\right]\right],\left[\left[M_{+}\right]\right]\right)=\lim _{k \rightarrow+\infty} \int_{0}^{n_{k}}\left[\mathcal{L}_{c}\left(t, \gamma_{t}^{k}, \dot{\gamma}_{t}^{k}\right)+\alpha(c)\right] \mathrm{d} t \\
\gamma_{0}^{k} \in\left[\left[M_{-}\right]\right], \quad \gamma_{n_{k}}^{k} \in\left[\left[M_{+}\right]\right] .
\end{array}\right.
$$

By Lemma 4.3 and the fact that $\Pi\left(M a t_{c}\right)$ is compact in $M_{\text {on }} \mathbf{Z}$, there are two integers $n_{k}^{\prime} \in\left[0, n_{k}\right]$ and $a_{k} \in \mathbf{Z}$ such that $\gamma_{n_{k}^{\prime}}^{k}-a_{k} \rightarrow N \in M a t_{c}$. Since $U^{-}$and $U^{+}$are $c$-dominated, we have that

$$
\begin{gathered}
U^{+}\left(\gamma_{n_{k}^{\prime}}^{k}\right)-U^{+}\left(M_{-}\right) \leq \int_{0}^{n_{k}^{\prime}}\left[\mathcal{L}_{c}\left(t, \gamma_{t}^{k}, \dot{\gamma}_{t}^{k}\right)+\alpha(c)\right] \mathrm{d} t \\
U^{-}\left(M_{+}\right)-U^{-}\left(\gamma_{n_{k}^{\prime}}^{n}\right) \leq \int_{n_{k}^{\prime}}^{n_{k}}\left[\mathcal{L}_{c}\left(t, \gamma_{t}^{k}, \dot{\gamma}_{t}^{k}\right)+\alpha(c)\right] \mathrm{d} t .
\end{gathered}
$$


We recall that $U^{-}$and $U^{+}$are $L_{\mathbf{Z}}^{2}$-invariant; adding the inequalities above, and letting $k \rightarrow+\infty$, we get by (4.7) that

$$
U^{-}\left(M_{+}\right)-U^{-}(N)+U^{+}(N)-U^{+}\left(M_{-}\right) \leq h_{\infty}\left(\left[\left[M_{-}\right]\right],\left[\left[M_{+}\right]\right]\right) .
$$

Since $N \in$ Mat $_{c}$, the definition of $\mathcal{D}$ implies that $U^{-}(N)=U^{+}(N)$, and the thesis follows.

Theorem 4.9. For $M_{-}, M_{+} \in$ Mon, we have that

$$
h_{\infty}\left(\left[\left[M_{-}\right]\right],\left[\left[M_{+}\right]\right]\right)=\sup _{\left(U^{-}, U^{+}\right) \in \mathcal{D}}\left[U^{-}\left(M_{-}\right)-U^{+}\left(M_{+}\right)\right] .
$$

Proof. By Lemma 4.8, we know that

$$
h_{\infty}\left(\left[\left[M_{-}\right]\right],\left[\left[M_{+}\right]\right]\right) \geq \sup _{\left(U^{-}, U^{+}\right) \in \mathcal{D}}\left[U^{-}\left(M_{-}\right)-U^{+}\left(M_{+}\right)\right] .
$$

To prove the opposite inequality, we see as in theorem 5.3.6 of [7] that, for all $M_{+} \in$ Mon, the function

$$
U_{M_{-}}^{-}: M_{+} \rightarrow h_{\infty}\left(\left[\left[M_{-}\right]\right],\left[\left[M_{+}\right]\right]\right)
$$

is a fixed point of $T_{1}^{-}$, while for all $M_{-} \in$ Mon, the function

$$
U_{M_{+}}^{+}: M_{-} \rightarrow h_{\infty}\left(\left[\left[M_{-}\right]\right],\left[\left[M_{+}\right]\right]\right)
$$

is a fixed point of $T_{-1}^{+}$. The reason for this is essentially the following: it is not hard to see that $Q: M_{+} \rightarrow h_{\infty}\left(\left[\left[M_{-}\right]\right],\left[\left[M_{+}\right]\right]\right)$is $c$-dominated; moreover, the curves $\gamma^{n}$ which minimize in the definition of $h_{n}\left(\left[\left[M_{-}\right]\right],\left[\left[M_{+}\right]\right]\right)$converge, up to subsequences, to a curve $\gamma$ calibrating for $Q$ on $(-\infty, 0]$; now the assertion follows by point 2) of Lemma 2.3.

Moreover, we can prove as in [7] that the conjugate function $U^{+}$of $U_{M_{-}}^{-}$ vanishes at $M_{-}$, while the conjugate function $U^{-}$of $U_{M_{+}}^{+}$vanishes at $M_{+}$. Indeed, since $U_{M_{-}}^{-}$is $c$-dominated, we can apply point 4) of theorem 4.2 and get that

$$
\begin{aligned}
U^{+} & \left(M_{-}\right)=\lim _{n \rightarrow+\infty}\left(T_{-n}^{+} U_{M_{-}}^{-}\right)\left(M_{-}\right) \\
= & \lim _{n \rightarrow+\infty} \max \left\{h_{\infty}\left(\left[\left[M_{-}\right]\right],\left[\left[\gamma_{n}\right]\right]\right)\right. \\
& \left.\quad-\int_{0}^{n}\left[\mathcal{L}_{c}(t, \gamma, \dot{\gamma})+\alpha(c)\right] \mathrm{d} t: \gamma_{0}=M_{-}\right\} .
\end{aligned}
$$

Let $\bar{\gamma}^{n}$ maximize in the formula above. For each $n$ we choose $\gamma^{n}$ minimal in the definition of $h_{n}\left([[M]],\left[\left[\bar{\gamma}_{n}^{n}\right]\right]\right)$; by compactness, there is $n_{k} \rightarrow+\infty$ such that $\left[\left[\gamma_{n_{k}}^{n_{k}}\right]\right] \rightarrow[[N]]$. By an argument like that of point 2) of Proposition 2.1, the functions $h_{n}$ can be shown to be $L$-Lipschitz in both variables, with the constant $L$ independent on $n$; this implies that $h_{\infty}$ is Lipschitz too. This, and the fact that $\gamma_{n_{k}}^{n_{k}} \rightarrow N$, imply the first and third equalities below; the last one follows by our choice of $\gamma^{n}$. 


$$
\begin{aligned}
\lim _{k \rightarrow+\infty} h_{\infty}\left(\left[\left[M_{-}\right]\right],\left[\left[\gamma_{n_{k}}^{n_{k}}\right]\right]\right) & =h_{\infty}\left(\left[\left[M_{-}\right]\right],[[N]]\right) \leq \liminf _{k \rightarrow+\infty} h_{n_{k}}\left(\left[\left[M_{-}\right]\right],[[N]]\right) \\
& =\liminf _{k \rightarrow+\infty} h_{n_{k}}\left(\left[\left[M_{-}\right]\right],\left[\left[\gamma_{n_{k}}^{n_{k}}\right]\right]\right) \\
& =\liminf _{k \rightarrow+\infty} \int_{0}^{n_{k}}\left[\mathcal{L}_{c}\left(t, \gamma^{n_{k}}, \dot{\gamma}^{n_{k}}\right)+\alpha(c)\right] \mathrm{d} t .
\end{aligned}
$$

The last two formulas imply that $U^{+}\left(M_{-}\right) \leq 0$. Since $\left(U_{M_{-}}^{-}, U^{+}\right) \in \mathcal{D}$, Lemma 4.8 implies the inequality below; the equality is the definition of $U_{M_{-}}^{-}$.

$h_{\infty}\left(\left[\left[M_{-}\right]\right],\left[\left[M_{+}\right]\right]\right) \geq U_{M_{-}}^{-}\left(M_{+}\right)-U^{+}\left(M_{-}\right)=h_{\infty}\left(\left[\left[M_{-}\right]\right],\left[\left[M_{+}\right]\right]\right)-U^{+}\left(M_{-}\right)$.

This implies that $U^{+}\left(M_{-}\right) \geq 0$, ending the proof that $U^{+}\left(M_{-}\right)=0$.

Since $U^{+}\left(M_{-}\right)=0$, we get the second equality below; the first one is the definition of $U_{M_{+}}^{-}$.

$$
h_{\infty}\left(\left[\left[M_{-}\right]\right],\left[\left[M_{+}\right]\right]\right)=U_{M_{-}}^{-}\left(M_{+}\right)=U_{M_{-}}^{-}\left(M_{+}\right)-U^{+}\left(M_{-}\right) .
$$

Since $\left(U_{M_{+}}^{-}, U^{+}\right) \in \mathcal{D}$, this yields the inequality opposite to (4.8).

As an immediate consequence, we can reunite Mather's definition in [13] with Fathi's definition, which we gave before theorem 4.7.

Theorem 4.10. $M \in \mathcal{A}_{c}$ iff $h_{\infty}(M, M)=0$.

We forego another check, i.e. that the Mañe set $\mathcal{M} N_{c}$ is the set of the $c$-minimal orbits.

\section{Fixed points and KAM}

Now we want to to look at the minimal orbits of $\mathcal{L}_{c}$ from another point of view, that of fixed point theory.

Definition. Let $\tilde{\mu}_{-1}, \tilde{\mu}_{1}$ be two Borel probability measures on $\mathbf{R}$, which we shall always suppose to be compactly supported. Actually, we shall only consider $\tilde{\mu}_{ \pm 1}$ of the form $\tilde{\mu}_{ \pm 1}=\left(\sigma_{ \pm 1}\right)_{\sharp} \nu_{0}$, with $\sigma_{ \pm 1} \in$ Mon, implying that $\tilde{\mu}_{ \pm 1}$ is supported in an interval of length 1 .

We denote by $\mathcal{M}_{1}\left(\tilde{\mu}_{1}, \tilde{\mu}_{2}\right)$ the space of the Borel probability measures on $[-1,1] \times \mathbf{R} \times \mathbf{R}$ which satisfy the following three points.

i)

$$
\int_{[-1,1] \times \mathbf{R} \times \mathbf{R}}(1+|v|) \mathrm{d} \mu(t, q, v)<+\infty .
$$

ii) Let $\pi:(t, q, v) \rightarrow t$. We ask that, if $\mu \in \mathcal{M}_{1}\left(\tilde{\mu}_{-1}, \tilde{\mu}_{1}\right)$, then $\pi_{\sharp} \mu=\frac{1}{2} \mathcal{L}^{1}$, where $\mathcal{L}^{1}$ denotes the Lebesgue measure on $[-1,1]$. In particular, $\mu$ is a probability measure, and can be disintegrated as $\mu=\frac{1}{2} \mathcal{L}^{1} \otimes \mu_{t}$, with $\mu_{t}$ a measure on $\mathbf{R} \times \mathbf{R}$. 
iii) We also ask that the elements $\mu$ of $\mathcal{M}_{1}\left(\tilde{\mu}_{-1}, \tilde{\mu}_{1}\right)$ are closed, i.e. for any $\phi \in C_{0}^{1}([-1,1] \times \mathbf{R})$, we have that

$$
\begin{aligned}
\int_{[-1,1] \times \mathbf{R} \times \mathbf{R}} \mathrm{d} \phi(t, q) \cdot(1, v) \mathrm{d} \mu(t, q, v)= & \frac{1}{2} \int_{\mathbf{R}} \phi(1, q) \mathrm{d} \tilde{\mu}_{1}(q) \\
& -\frac{1}{2} \int_{\mathbf{R}} \phi(-1, q) \mathrm{d} \tilde{\mu}_{-1}(q) .
\end{aligned}
$$

In [4], the elements of $\mathcal{M}_{1}\left(\tilde{\mu}_{-1}, \tilde{\mu}_{1}\right)$ are called the transport measures.

Point $i$ ) above essentially says that the integral on the left of (5.1) converges; point $i i i)$ says that $\mu$ has "boundary values" $\tilde{\mu}_{-1}$ at $t=-1$, and $\tilde{\mu}_{1}$ at $t=1$. As an example, consider $\sigma \in C^{1}\left([-1,1], L^{2}(I)\right)$; if we define $\tilde{\mu}_{ \pm 1}=\left(\sigma_{ \pm 1}\right)_{\sharp} \nu_{0}, \mu_{t}=\left(\sigma_{t}, \dot{\sigma}_{t}\right)_{\sharp} \nu_{0}$ and $\mu=\frac{1}{2} \mathcal{L}^{1} \otimes \mu_{t}$, then it is easy to check that $\mu \in \mathcal{M}_{1}\left(\tilde{\mu}_{-1}, \tilde{\mu}_{1}\right)$. We saw above that $\tilde{\mu}_{ \pm 1}$ are supported in an interval of length 1 .

It is well-known $([4])$ that we can endow $\mathcal{M}_{1}\left(\tilde{\mu}_{-1}, \tilde{\mu}_{1}\right)$ with a distance $d$ (called a Kantorovich-Rubinstein distance) with the following property: $d\left(\mu_{n}, \mu\right) \rightarrow 0$ if, for any $\phi \in C([-1,1] \times \mathbf{R} \times \mathbf{R})$ such that

$$
\sup _{(t, q, v)} \frac{|\phi(t, q, v)|}{1+|v|}<+\infty
$$

we have that

$$
\int_{[-1,1] \times \mathbf{R} \times \mathbf{R}} \phi(t, q, v) \mathrm{d} \mu_{n}(t, q, v) \rightarrow \int_{[-1,1] \times \mathbf{R} \times \mathbf{R}} \phi(t, q, v) \mathrm{d} \mu(t, q, v) .
$$

By [4], $d$ turns $\mathcal{M}_{1}\left(\tilde{\mu}_{1}, \tilde{\mu}_{2}\right)$ into a complete metric space.

It is a standard consequence of $i$ ), $i i$ ), and $i i i$ ) above (the proof is akin to Lemma 8.1.2 of [2]) that, for any choice of the $C^{1}$ function $\phi$, the function

$$
: t \rightarrow \int_{\mathbf{R} \times \mathbf{R}} \phi(q) \mathrm{d} \mu_{t}(q, v)
$$

is absolutely continuous. In particular, the function

$$
W_{\mu}(t, x)=\int_{\mathbf{R} \times \mathbf{R}} W(x-y) \mathrm{d} \mu_{t}(y, v)
$$

is continuous in $t$. Since we are supposing that $W \in C^{2}\left(S^{1}\right)$, differentiating under the integral sign we get that $W_{\mu} \in C\left([-1,1], C^{2}\left(S^{1}\right)\right)$; actually, we get that $\left\|W_{\mu}\right\|_{C\left([-1,1], C^{2}\left(S^{1}\right)\right)}$ is bounded by a constant independent on $\mu$. This prompts us to define, for $\mu \in \mathcal{M}_{1}\left(\tilde{\mu}_{-1}, \tilde{\mu}_{1}\right)$,

$$
L_{\mu, c}:[-1,1] \times S^{1} \times \mathbf{R} \rightarrow \mathbf{R}
$$

by

$$
L_{\mu, c}(t, x, \dot{x})=\frac{1}{2}|\dot{x}|^{2}-c \dot{x}-V(t, x)-W_{\mu}(t, x) .
$$

An important case is that in which $\mu_{t}=\left(\sigma_{t}, \dot{\sigma}_{t}\right)_{\sharp} \nu_{0}$, with $\sigma c$-minimal; we saw in Sect. 3 that, in this case, $\sigma \in C^{2}\left(\mathbf{R}, L^{2}(I)\right)$; actually, there is $C_{1}>$ 0 such that, for any $c$-minimal $\sigma,\|\sigma\|_{C^{2}\left(\mathbf{R}, L^{2}(I)\right)} \leq C_{1}$; as a consequence, $\left\|W_{\mu}\right\|_{C^{2}\left([-1,1] \times S^{1}\right)} \leq C_{2}$, with $C_{2}$ not depending on the $c$-minimal $\sigma$. 
To avoid proving theorems about compactness, a small haircut on transfer measures is necessary.

Definition. We define $A\left(\tilde{\mu}_{-1}, \tilde{\mu}_{1}\right)$ as the smallest $R$ for which $B_{R}:=[-R, R]$ contains the supports of both $\tilde{\mu}_{-1}$ and $\tilde{\mu}_{1}$.

For $R \geq A\left(\tilde{\mu}_{-1}, \tilde{\mu}_{1}\right)$, let us call $\mathcal{M}_{1}^{R}\left(\tilde{\mu}_{-1}, \tilde{\mu}_{1}\right)$ the set of the elements of $\mathcal{M}_{1}\left(\tilde{\mu}_{-1}, \tilde{\mu}_{1}\right)$ which are supported in $[-1,1] \times B_{R} \times B_{R}$. Note that $\mathcal{M}_{1}^{R}\left(\tilde{\mu}_{-1}, \tilde{\mu}_{1}\right)$ is a compact subset of $\mathcal{M}_{1}\left(\tilde{\mu}_{-1}, \tilde{\mu}_{1}\right)$. It follows from [3] that, for $R$ large enough, $\mathcal{M}_{1}^{R}\left(\tilde{\mu}_{-1}, \tilde{\mu}_{1}\right)$ is not empty.

Lemma 5.1. Let $\delta \in \mathcal{M}_{1}\left(\tilde{\mu}_{-1}, \tilde{\mu}_{1}\right)$, and let $K$ be so large that $\mathcal{M}_{1}^{K}\left(\tilde{\mu}_{-1}, \tilde{\mu}_{1}\right)$ is not empty.

1) Then, there is $\bar{\mu} \in \mathcal{M}_{1}^{K}\left(\tilde{\mu}_{-1}, \tilde{\mu}_{1}\right)$ such that

$$
\begin{aligned}
& \int_{[-1,1] \times \mathbf{R} \times \mathbf{R}} L_{\delta, c}(t, q, v) \mathrm{d} \bar{\mu}(t, q, v) \\
& \quad=\inf \left\{\int_{[-1,1] \times \mathbf{R} \times \mathbf{R}} L_{\delta, c}(t, q, v) \mathrm{d} \mu(t, q, v): \mu \in \mathcal{M}_{1}^{K}\left(\tilde{\mu}_{-1}, \tilde{\mu}_{1}\right)\right\} .
\end{aligned}
$$

2) The set of all the measures $\bar{\mu}$ which satisfy the formula above is a compact, convex set $C_{\delta}$ of $\mathcal{M}_{1}^{K}\left(\tilde{\mu}_{-1}, \tilde{\mu}_{1}\right)$.

3) There is $R>0$, depending on $A\left(\tilde{\mu}_{-1}, \tilde{\mu}_{1}\right)$ but not on $\delta$, such that, for $K \geq R, C_{\delta}$ does not depend on $K$.

Proof. We only sketch the standard proof of this lemma. We saw above that $\mathcal{M}_{1}^{K}\left(\tilde{\mu}_{-1}, \tilde{\mu}_{1}\right)$ is compact; thus, point 1$)$ is a standard consequence of the fact that the functional

$$
: \mu \rightarrow \int_{[-1,1] \times \mathbf{R} \times \mathbf{R}} L_{\delta, c}(t, q, v) \mathrm{d} \mu(t, q, v)
$$

is 1. s. c. (see for instance [4]). We prove point 2); since $\mathcal{M}_{1}^{K}\left(\tilde{\mu}_{-1}, \tilde{\mu}_{1}\right)$ is compact, it suffices to prove that $C_{\delta}$ is convex and closed; this is again a consequence of the fact that the map displayed above is linear and l.s.c..

As for point 3 ), we recall the fact, proven in [3], that any minimal $\bar{\mu}$ is supported in a set of orbits $q$ minimal for $L_{\delta, c}$; thus, the thesis follows if we prove that there is $R>0$, independent on $\delta$, such that any minimal $q$, connecting a point in the support of $\tilde{\mu}_{-1}$ with another in the support of $\tilde{\mu}_{1}$, satisfies $(q(t), \dot{q}(t)) \in B_{R} \times B_{R}$. Since $q( \pm 1)$ lie in the supports of $\tilde{\mu}_{ \pm 1}$, i.e. in the interval $B_{A\left(\tilde{\mu}_{-1}, \tilde{\mu}_{1}\right)}$, it suffices a bound on $\dot{q}$ : we shall prove that $q$ satisfies $|\dot{q}(t)| \leq C$ for a constant $C$ depending only on $A\left(\tilde{\mu}_{-1}, \tilde{\mu}_{1}\right)$.

Actually, with the same argument of Lemma 3.4, we can prove that there is $C>0$ such that, if $q$ is minimal for $L_{\delta, c}$ and connects two points in $B_{A\left(\tilde{\mu}_{-1}, \tilde{\mu}_{1}\right)}$, then $|\dot{q}| \leq C$. The constant $C$ depends only on $\left\|V+W_{\delta}\right\|_{C\left([-1,1], C^{2}\left(\mathbf{T}^{p}\right)\right)}$ (which we know to be bounded independently on $\delta$ ) and on $A\left(\tilde{\mu}_{-1}, \tilde{\mu}_{1}\right)$ (the maximal distance of the points to be connected), ending the proof.

Definition. We settle a bit of notation: from now on, $R$ will be the constant of point 3 ) of the lemma above. 
If $\mu \in \mathcal{M}_{1}^{R}\left(\tilde{\mu}_{-1}, \tilde{\mu}_{1}\right)$ is minimal in point 1$)$ of Lemma 5.1 , we call it a minimal transfer measure for $L_{\delta, c}$.

Let $\mathcal{C}$ denote the class of all closed, convex subsets of $\mathcal{M}_{1}^{R}\left(\tilde{\mu}_{-1}, \tilde{\mu}_{1}\right)$; by point 2) of Lemma 5.1, we have a map

$$
\Phi: \mathcal{M}_{1}^{R}\left(\tilde{\mu}_{-1}, \tilde{\mu}_{1}\right) \rightarrow \mathcal{C}
$$

which brings $\delta$ into the set $C_{\delta}$ of minimal transfer measures for $L_{\delta, c}$.

We assert that the set valued map $\Phi$ is upper semicontinuous, i.e. that, if $\delta_{n} \rightarrow \delta$, if $\mu_{n}$ is minimal for $\mathcal{L}_{\delta_{n}, c}$ and $\mu_{n} \rightarrow \mu$, then $\mu$ is minimal for $\mathcal{L}_{\delta, c}$. We sketch the standard proof of this; for starters, since $\mathcal{M}_{1}^{R}\left(\tilde{\mu}_{-1}, \tilde{\mu}_{1}\right)$ is closed in $\mathcal{M}_{1}\left(\tilde{\mu}_{-1}, \tilde{\mu}_{1}\right)$, we get that $\mu \in \mathcal{M}_{1}^{R}\left(\tilde{\mu}_{-1}, \tilde{\mu}_{1}\right)$. It is proven in [3] that the function

$$
: \mu \rightarrow \int_{[-1,1] \times \mathbf{R} \times \mathbf{R}}\left[\frac{1}{2}|v|^{2}-c \cdot v-V(t, q)\right] \mathrm{d} \mu(t, q, v)
$$

is l. s. c.. Moreover, since $\delta_{n} \rightarrow \delta$, we have that $W_{\delta_{n}} \rightarrow W_{\delta}$ uniformly; these two facts imply that

$$
\int_{[-1,1] \times \mathbf{R} \times \mathbf{R}} L_{\delta, c}(t, q, v) \mathrm{d} \mu(t, q, v) \leq \liminf _{n \rightarrow+\infty} \int_{[-1,1] \times \mathbf{R} \times \mathbf{R}} L_{\delta_{n}, c}(t, q, v) \mathrm{d} \mu_{n}(t, q, v) .
$$

Let us suppose by contradiction that $\mu$ is not a minimal transfer measure for $L_{\delta, c}$; by the formula above, this means that there is $\bar{\mu} \in \mathcal{M}_{1}^{R}\left(\tilde{\mu}_{-1}, \tilde{\mu}_{1}\right)$ such that

$$
\int_{[-1,1] \times \mathbf{R} \times \mathbf{R}} L_{\delta, c}(t, q, v) \mathrm{d} \bar{\mu}(t, q, v)<\liminf _{n \rightarrow+\infty} \int_{[-1,1] \times \mathbf{R} \times \mathbf{R}} L_{\delta_{n}, c}(t, q, v) \mathrm{d} \mu_{n}(t, q, v) .
$$

Since $W_{\delta_{n}} \rightarrow W_{\delta}$ uniformly, the formula above implies that, for $n$ large enough, the inequality below holds.

$$
\begin{aligned}
& \int_{[-1,1] \times \mathbf{R} \times \mathbf{R}} L_{\delta_{n}, c}(t, q, v) \mathrm{d} \bar{\mu}(t, q, v) \\
& \quad=\int_{[-1,1] \times \mathbf{R} \times \mathbf{R}} L_{\delta, c}(t, q, v) \mathrm{d} \bar{\mu}(t, q, v)-\int_{[-1,1] \times \mathbf{R} \times \mathbf{R}}\left[W_{\delta_{n}}-W_{\delta}\right] \mathrm{d} \bar{\mu}(t, q, v) \\
& \quad<\int_{[-1,1] \times \mathbf{R} \times \mathbf{R}} L_{\delta_{n}, c}(t, q, v) \mathrm{d} \mu_{n}(t, q, v) .
\end{aligned}
$$

This contradicts the fact that $\mu_{n}$ is minimal for $L_{\delta_{n}, c}$, i.e. that $\mu_{n} \in \Phi\left(\delta_{n}\right)$.

Since $\mathcal{M}_{1}^{R}\left(\tilde{\mu}_{1}, \tilde{\mu}_{2}\right)$ is compact and the map $\Phi$ is upper semicontinuous, we can apply the Ky Fan theorem [11] and find $\mu$ such that $\mu \in \Phi(\mu)$; let us gather in a set $S$ the measures $\mu$ for which $\mu \in \Phi(\mu)$. Again from the fact that $\Phi$ is u. s. c., it follows that $S$ is a closed set of $\mathcal{M}_{1}^{R}\left(\tilde{\mu}_{-1}, \tilde{\mu}_{1}\right)$; thus, it is compact, and we can find $\bar{\mu} \in S$ such that

$$
\begin{aligned}
a\left(\tilde{\mu}_{1}, \tilde{\mu}_{2}\right): & =\int_{[-1,1] \times \mathbf{R} \times \mathbf{R}} L_{\frac{1}{2} \bar{\mu}, c}(t, x, v) \mathrm{d} \bar{\mu}(t, x, v) \\
& =\inf _{\mu \in S} \int_{[-1,1] \times \mathbf{R} \times \mathbf{R}} L_{\frac{1}{2} \mu, c}(t, x, v) \mathrm{d} \mu(t, x, v) .
\end{aligned}
$$

We need a definition. 
Definition. We shall say that $\sigma$ is minimal for $\mathcal{L}_{c}$ if it is minimal among all A. C. curves $\gamma$ with $\gamma_{ \pm 1}=\sigma_{ \pm 1}$. This is a weaker notion that the $c$-minimality of Sect. 1 , where we only required that $\gamma_{ \pm 1}-\sigma_{ \pm 1} \in L_{\mathbf{Z}}^{2}$. In other words, now we are considering particles on $\mathbf{R}$, not on $S^{1}$.

The next lemma gives us the relation between the minimal transfer measures $\mu$ and the minimal paths $\sigma$; it can be seen as a different proof of formula (12) of [8]. Note the quirk of notation: in the definition of $a\left(\tilde{\mu}_{1}, \tilde{\mu}_{2}\right)$ we are minimizing the integral of $L_{\frac{1}{2} \mu, c}$, but over all the minimal transfer measures $\mu$ for $L_{\mu, c}$. We shall see the reasons for this factor $\frac{1}{2}$ in the proof below.

Lemma 5.2. 1) Let $\bar{\sigma} \in A C_{m o n}(-1,1)$ be minimal for $\mathcal{L}_{c}$, and let us consider the two measures on $\mathbf{R} \tilde{\mu}_{-1}=\left(\bar{\sigma}_{-1}\right)_{\sharp} \nu_{0}, \tilde{\mu}_{1}=\left(\bar{\sigma}_{1}\right)_{\sharp} \nu_{0}$. Then,

$$
a\left(\tilde{\mu}_{-1}, \tilde{\mu}_{1}\right)=\int_{-1}^{1} \mathcal{L}_{c}\left(t, \bar{\sigma}_{t}, \dot{\bar{\sigma}}_{t}\right) \mathrm{d} t
$$

2) Moreover, if $a\left(\tilde{\mu}_{-1}, \tilde{\mu}_{1}\right)$ is attained on $\mu$, then $\mu$ is induced by a minimal parametrization $\sigma_{t}$; vice-versa, if $\sigma_{t}$ is a minimal parametrization, then $a\left(\tilde{\mu}_{-1}, \tilde{\mu}_{1}\right)$ is attained on the measure induced by $\sigma_{t}$.

Proof. We begin with point 1). For $M_{-1}, M_{1} \in$ Mon, we define

$$
b\left(M_{-1}, M_{1}\right)=\min \left\{\int_{-1}^{1} \mathcal{L}_{c}\left(t, \sigma_{t}, \dot{\sigma}_{t}\right) \mathrm{d} t: \sigma_{-1}=M_{-1}, \quad \sigma_{1}=M_{1}\right\} .
$$

Thus, we have to prove that

$$
b\left(\sigma_{-1}, \sigma_{1}\right)=a\left(\tilde{\mu}_{1}, \tilde{\mu}_{2}\right) .
$$

We begin to show that

$$
b\left(\bar{\sigma}_{-1}, \bar{\sigma}_{1}\right) \leq a\left(\tilde{\mu}_{1}, \tilde{\mu}_{2}\right) .
$$

Let $\mu$ minimize in the definition of $a\left(\tilde{\mu}_{-1}, \tilde{\mu}_{1}\right)$; then, $\mu \in S$, which implies that $\mu$ is a minimal transfer measure for $L_{\mu, c}$.

We assert that there is a parametrization $\sigma \in A C_{m o n}(-1,1)$ such that $\mu=\frac{1}{2} \mathcal{L}^{1} \otimes\left(\sigma_{t}, \dot{\sigma}_{t}\right)_{\sharp} \nu_{0}$; note that this implies, by the definition of push-forward, that

$$
\int_{-1}^{1} \mathcal{L}_{c}\left(t, \sigma_{t}, \dot{\sigma}_{t}\right) \mathrm{d} t=\int_{[-1,1] \times \mathbf{R} \times \mathbf{R}} L_{\frac{1}{2} \mu, c}(t, x, v) \mathrm{d} \mu(t, x, v)
$$

from which (5.3) follows. Once we shall have proven that $b\left(\sigma_{-1}, \sigma_{1}\right)=$ $a\left(\tilde{\mu}_{-1}, \tilde{\mu}_{1}\right)$, the formula above will yield part of point 2$)$, i.e. that the minimal measure $\mu$ is induced by a minimal parametrization $\sigma$.

The proof of the assertion is essentially contained in Sect. 4.2 of [3], which says that, if $\mu$ is a minimal transfer measure for a Lagrangian, say $L_{\mu, c}$, then $\mu$ is supported on a bunch of minimal orbits of $L_{\mu, c}$, which can be easily parametrized.

More precisely, let $\psi_{s}^{t}$ be the Euler-Lagrange flow of $L_{\mu, c}: \psi_{s}^{t}$ brings an initial condition $(x, v)$ at time $s$ into its evolution at time $t$. By Sect. 4.2 of [3], there is a probability measure $\tilde{\mu}_{0}$ on $\mathbf{R}$ and a Lipschitz function $v: \mathbf{R} \rightarrow \mathbf{R}$ such that, setting 


$$
\mu_{t}=\left(\psi_{0}^{t}\right)_{\sharp}(i d, v)_{\sharp} \tilde{\mu}_{0},
$$

then $\mu=\frac{1}{2} \mathcal{L}^{1} \otimes \mu_{t}$. Take the monotone map $\sigma_{0}$ which brings $\nu_{0}$, the Lebesgue measure on the parameter space $[0,1]$, into $\mu_{0}$ and set

$$
\left(\sigma_{t}, \dot{\sigma}_{t}\right)=\psi_{0}^{t} \circ(i d, v) \circ \sigma_{0}
$$

By the two formulas above, it is immediate that $\mu_{t}=\left(\sigma_{t}, \dot{\sigma}_{t}\right)_{\sharp} \nu_{0}$, and this proves the assertion.

We prove the inequality opposite to (5.3). Let $b\left(M_{-1}, M_{1}\right)$ be attained on $\sigma$; let $\tilde{\mu}_{ \pm 1}=\left(\sigma_{ \pm 1}\right)_{\sharp} \nu_{0}, \mu_{t}=\left(\sigma_{t}, \dot{\sigma}_{t}\right)_{\sharp} \nu_{0}$ and $\mu=\frac{1}{2} \mathcal{L}^{1} \otimes \mu_{t}$.

We begin to prove that, for $\nu_{0}$ a.e. $x$, the orbit $: t \rightarrow \sigma_{t} x$ is minimal, for fixed endpoints. To show this, let $x_{0}$ be a Lebesgue point for both maps $: x \rightarrow\left(\sigma_{ \pm 1} x, \dot{\sigma}_{ \pm 1} x\right)$, i.e. let

$$
\lim _{\epsilon \rightarrow 0+} \frac{1}{2 \epsilon} \int_{x_{0}-\epsilon}^{x_{0}+\epsilon}\left(\sigma_{ \pm 1} x, \dot{\sigma}_{ \pm 1} x\right) \mathrm{d} x=\left(\sigma_{ \pm 1} x_{0}, \dot{\sigma}_{ \pm 1} x_{0}\right) .
$$

We write

$$
\begin{aligned}
& \int_{-1}^{1} \mathrm{~d} t\left[\int_{I} \frac{1}{2}\left|\dot{\sigma}_{t} x\right|^{2} \mathrm{~d} x-\int_{I} V\left(t, \sigma_{t} x\right) \mathrm{d} x-\frac{1}{2} \int_{I \times I} W\left(\sigma_{t} x-\sigma_{t} y\right) \mathrm{d} x \mathrm{~d} y\right] \\
& =\int_{-1}^{1} \mathrm{~d} t\left[\int_{I \backslash\left[x_{0}-\epsilon, x_{0}+\epsilon\right]} \frac{1}{2}\left|\dot{\sigma}_{t} x\right|^{2} \mathrm{~d} x-\int_{I \backslash\left[x_{0}-\epsilon, x_{0}+\epsilon\right]} V\left(t, \sigma_{t} x\right) \mathrm{d} x\right. \\
& \left.\quad-\frac{1}{2} \int_{\left(I \backslash\left[x_{0}-\epsilon, x_{0}+\epsilon\right]\right)^{2}} W\left(\sigma_{t} x-\sigma_{t} y\right) \mathrm{d} x \mathrm{~d} y\right] \\
& +\int_{-1}^{1} \mathrm{~d} t\left[\int_{\left[x_{0}-\epsilon, x_{0}+\epsilon\right]} \frac{1}{2}\left|\dot{\sigma}_{t} x\right|^{2} \mathrm{~d} x-\int_{\left[x_{0}-\epsilon, x_{0}+\epsilon\right]} V\left(t, \sigma_{t} x\right) \mathrm{d} x\right. \\
& \quad-\int_{\left[x_{0}-\epsilon, x_{0}+\epsilon\right] \times\left(I \backslash\left[x_{0}-\epsilon, x_{0}+\epsilon\right]\right)} W\left(\sigma_{t} x-\sigma_{t} y\right) \mathrm{d} x \mathrm{~d} y \\
& \left.+\frac{1}{2} \int_{\left[x_{0}-\epsilon, x_{0}+\epsilon\right]^{2}} W\left(\sigma_{t} x-\sigma_{t} y\right) \mathrm{d} x \mathrm{~d} y\right]
\end{aligned}
$$

Note that, if $x \in\left[x_{0}-\epsilon, x_{0}+\epsilon\right]$, the trajectory $: t \rightarrow \sigma_{t} x$ doesn't appear in (5.4a) of the formula above, but only in $(5.4 \mathrm{~b})$; thus, the curve parametrized by $\left.\sigma_{t}\right|_{\left[x_{0}-\epsilon, x_{0}+\epsilon\right]}$ minimizes the integral in $(5.4 \mathrm{~b})$ for fixed boundary conditions. We assert that this implies that $: t \rightarrow \sigma_{t} x_{0}$ is minimal for $L_{\mu, c}$, endpoints fixed.

Indeed, let us suppose by contradiction that there is : $t \rightarrow q(t)$, with the same extrema, such that

$$
\int_{-1}^{1} L_{\mu, c}(t, q(t), \dot{q}(t)) \mathrm{d} t<\int_{-1}^{1} L_{c, \mu}\left(t, \sigma_{t} x_{0}, \dot{\sigma}_{t} x_{0}\right) \mathrm{d} t .
$$

For $\epsilon>0$, define $\gamma=\gamma(\epsilon)$ as the largest one among $\sigma_{1}\left(x_{0}+\epsilon\right)-\sigma_{1}\left(x_{0}\right)$, $\sigma_{1}\left(x_{0}\right)-\sigma_{1}\left(x_{0}-\epsilon\right), \sigma_{-1}\left(x_{0}+\epsilon\right)-\sigma_{1}\left(x_{0}\right)$ and $\sigma_{-1}\left(x_{0}\right)-\sigma_{1}\left(x_{0}-\epsilon\right)$; since $x_{0}$ is a Lebesgue point of $\sigma_{ \pm 1}$, we have that $\gamma \rightarrow 0$ as $\epsilon \rightarrow 0$. 
For $x \in\left[x_{0}-\epsilon, x_{0}+\epsilon\right]$, we define

$$
\tilde{\sigma}_{t} x= \begin{cases}\frac{t+1-\gamma}{-\gamma} \sigma_{-1} x+\frac{t+1}{\gamma} q(t) & -1 \leq t \leq-1+\gamma \\ q(t) & -1+\gamma \leq t \leq 1-\gamma \\ \frac{t-1}{\gamma} q(t)+\frac{t-1+\gamma}{\gamma} \sigma_{1} x & 1-\gamma \leq t \leq 1\end{cases}
$$

If $x \notin\left[x_{0}-\epsilon, x_{0}+\epsilon\right]$, we set $\tilde{\sigma}_{t} x=\sigma_{t} x$. Since $\sigma_{t}$ solves $(O D E)_{L a g}$, for $\nu_{0}$ a.e. $x$ $: t \rightarrow \sigma_{t} x$ is an orbit of $L_{\mu, c}$; in particular, it is $C^{2}$ and depends continuously, in the $C^{2}$ topology, from the initial condition $\left(\sigma_{0} x, \dot{\sigma}_{0} x\right)$. Using this, the fact that $x_{0}$ is a Lebesgue point of $: x \rightarrow\left(\sigma_{t} x, \dot{\sigma}_{t} x\right)$ and formula (5.5), it is easy to see that

$$
\begin{aligned}
\int_{-1}^{1} \mathrm{~d} t & {\left[\int_{\left[x_{0}-\epsilon, x_{0}+\epsilon\right]} \frac{1}{2}\left|\dot{\tilde{\sigma}}_{t} x\right|^{2} \mathrm{~d} x-\int_{\left[x_{0}-\epsilon, x_{0}+\epsilon\right]} V\left(t, \tilde{\sigma}_{t} x\right) \mathrm{d} x\right.} \\
& -\int_{\left[x_{0}-\epsilon, x_{0}+\epsilon\right] \times\left(I \backslash\left[x_{0}-\epsilon, x_{0}+\epsilon\right]\right)} W\left(\tilde{\sigma}_{t} x-\tilde{\sigma}_{t} y\right) \mathrm{d} x \mathrm{~d} y \\
& \left.+\frac{1}{2} \int_{\left[x_{0}-\epsilon, x_{0}+\epsilon\right]^{2}} W\left(\tilde{\sigma}_{t} x-\tilde{\sigma}_{t} y\right) \mathrm{d} x \mathrm{~d} y\right] \\
< & \int_{-1}^{1} \mathrm{~d} t\left[\int_{\left[x_{0}-\epsilon, x_{0}+\epsilon\right]} \frac{1}{2}\left|\dot{\sigma}_{t} x\right|^{2} \mathrm{~d} x-\int_{\left[x_{0}-\epsilon, x_{0}+\epsilon\right]} V\left(t, \sigma_{t} x\right) \mathrm{d} x\right. \\
& -\int_{\left[x_{0}-\epsilon, x_{0}+\epsilon\right] \times\left(I \backslash\left[x_{0}-\epsilon, x_{0}+\epsilon\right]\right)} W\left(\sigma_{t} x-\sigma_{t} y\right) \mathrm{d} x \mathrm{~d} y \\
& \left.+\frac{1}{2} \int_{\left[x_{0}-\epsilon, x_{0}+\epsilon\right]^{2}} W\left(\sigma_{t} x-\sigma_{t} y\right) \mathrm{d} x \mathrm{~d} y\right]
\end{aligned}
$$

contradicting the fact that $\left.\sigma_{t}\right|_{\left[x_{0}-\epsilon, x_{0}+\epsilon\right]}$ minimizes the integral in $(5.4 \mathrm{~b})$.

Thus, for $\nu_{0}$ a.e. $x$ the orbit $: t \rightarrow \sigma_{t} x$ is minimal for $L_{\mu, c}$; note that we have lost the factor $\frac{1}{2}$ in the potential of $\mathcal{L}_{c}$ (the reason for this in in formula (5.4a)), and this explains the quirk of notation mentioned above.

The fact that, for $\nu_{0}$ a.e. $x$ the orbit $: t \rightarrow \sigma_{t} x$ is minimal for $L_{\mu, c}$, together with the fact that the map $: \sigma_{-1} x \rightarrow \sigma_{1} x$ brings $\tilde{\mu}_{-1}$ into $\tilde{\mu}_{1}$, implies by [3] that $\mu=\frac{1}{2} \mathcal{L}^{1} \otimes \mu_{t}$ is a minimal transfer measure. Actually, this is a standard fact of transport theory: in dimension one, if minimal characteristics cannot intersect, then the unique monotone map bringing $\tilde{\mu}_{-1}$ into $\tilde{\mu}_{1}$ is a minimal transfer map. As a consequence, we get the other half of point 2): if $\sigma$ is minimal, then the measure induced by $\sigma$ is a minimal transfer measure.

Proposition 5.3. Let $\sigma \in A C_{m o n}(\mathbf{R})$ be c-minimal. Let us consider the set

$$
A=\left\{\left(t, \sigma_{t} x\right): t \in \mathbf{R}, \quad x \in I\right\} \subset \mathbf{R} \times S^{1} .
$$

Then, the map

$$
\Gamma: A \rightarrow \mathbf{R}, \quad \Gamma:\left(t, \sigma_{t} x\right) \rightarrow \dot{\sigma}_{t} x
$$

is Lipschitz. 
Proof. Since $\sigma$ is $c$-minimal, it is also minimal for $\mathcal{L}_{c}$ in the weaker sense defined above; in particular, Lemma 5.2 holds.

Let $\tilde{\mu}_{t}=\left(\sigma_{t}\right)_{\sharp} \nu_{0}$, and let $\mu=\frac{1}{2} \mathcal{L}^{1} \otimes\left(\sigma_{t}, \dot{\sigma}_{t}\right)_{\sharp} \nu_{0}$; by Lemma $5.2, \mu$ is a minimal transport measure for $L_{\mu, c}$ between $\tilde{\mu}_{k-1}$ and $\tilde{\mu}_{k+1}, k \in \mathbf{Z}$. Now we can apply the addendum in Section 1.3 of [3], which says that $\Gamma$ is $L$-Lipschitz for $t \in\left[k-\frac{1}{2}, k+\frac{1}{2}\right]$; the Lipschitz constant $L$ depends only on the $C^{2}$ norm of $V$ and $W_{\mu}$, and on the radius of the smallest ball containing the supports of $\tilde{\mu}_{k-1}$ and $\tilde{\mu}_{k+1}$. Since we are free to translate by an integer, $L$ depends on the diameter of the union of the supports of $\tilde{\mu}_{k-1}$ and $\tilde{\mu}_{k+1}$. We note that $V$ is fixed, while $\left\|W_{\mu}\right\|_{C^{2}(\mathbf{R} \times \mathbf{R})}$ is bounded, since $W \in C^{2}\left(S^{1}\right)$ and the $C^{2}$ norm of $\sigma_{t}$, which solves $(O D E)_{\text {Lag }}$, is bounded (we saw in Lemma 3.4 that $\sup _{t \in \mathbf{R}}\left\|\dot{\sigma}_{t}\right\|$ is bounded). Since $\sigma_{t}$ belongs to Mon and has bounded speed in $L^{2}(I)$, the diameter of the union of the supports of $\tilde{\mu}_{k-1}$ and $\tilde{\mu}_{k+1}$ is bounded, uniformly in $k$. Thus, the Lipschitz constant $L$ of $\Gamma$ on $\left[k-\frac{1}{2}, k+\frac{1}{2}\right]$ does not depend on $k$, and the thesis follows.

We want to study the regularity of periodic minimal measures of irrational rotation number. We consider the Lagrangian

$$
\mathcal{L}^{\epsilon}(t, \sigma, v)=\frac{1}{2}\|v\|^{2}-\epsilon \mathcal{V}(t, \sigma)-\epsilon \mathcal{W}(\sigma)
$$

where $\mathcal{V}$ and $\mathcal{W}$ are defined as in Sect. 1 ; the only difference is that we ask that the potentials $V$ and $W$ are $C^{k}$ for some large $k$ which we shall determine in the following.

We want to study

$$
\min \left\{\int_{0}^{1} \mathcal{L}^{\epsilon}\left(t, \sigma_{t}, \dot{\sigma}_{t}\right) \mathrm{d} t: \sigma \in \mathcal{P}_{\omega}\right\}
$$

where by $\mathcal{P}_{\omega}$ we denote the set of those $\sigma \in A C_{\text {mon }}([0,1])$ such that

- $\sigma_{0} \simeq \sigma_{1}$ in the sense of Sect. 1 , or $\sigma$ projects to a periodic curve on $\mathbf{S}$; in other words,

$$
\left(\sigma_{0}\right)_{\sharp} \nu_{0}=\left(\sigma_{1}\right)_{\sharp} \nu_{0} .
$$

- Moreover, we ask that the rotation number of $\sigma$ is $\omega$; in other words,

$$
\int_{0}^{1}\left\langle 1, \dot{\sigma}_{t}\right\rangle \mathrm{d} t=\omega .
$$

We note that $\mathcal{P}_{\omega}$ is closed in $A C_{m o n}([0,1])$ : we forego the easy proof that $(5.7)$ and (5.8) are closed under uniform convergence. Moreover, $\mathcal{P}_{\omega}$ is not empty, since it is easy to see that $\sigma_{t} x=x+\omega t$ is periodic in $\mathbf{S}$ (actually, it is constant) and has rotation number $\omega$. As a consequence, we were justified in writing min in (5.6).

Let $\gamma, \tau>0$; we say that $\omega \in \mathbf{R}$ is $(\gamma, \tau)$-diophantine if

$$
|\omega q-p| \geq \frac{\gamma}{q^{\tau}} \quad \text { if } \quad(q, p) \in(\mathbf{N} \backslash\{0\}) \times \mathbf{Z}
$$

We want to prove the following. 
Theorem 5.4. Let $\omega$ be $(\gamma, \tau)$-diophantine, and let $\sigma$ minimize in (5.6). Then, there is $k_{0}(\gamma, \tau)>0$ such that, if $V$ and $W$ are $C^{k}$ with $k \geq k_{0}(\gamma, \tau)$ and $\epsilon$ is small enough, the measure on $[0,1] \times S^{1} \times \mathbf{R}$ given by $\mu:=\mathcal{L}^{1} \otimes\left(\sigma_{t}, \dot{\sigma}_{t}\right)_{\sharp} \nu_{0}$ is the push-forward of the Lebesgue measure on $S^{1} \times S^{1}$ by a $C^{1} \operatorname{map} \Phi:(t, x) \rightarrow$ $\left(t, \phi_{1}(t, x), \phi_{2}(t, x)\right)$.

Proof. Let $\sigma$ be minimal in (5.6), and let $\mu=\mathcal{L}^{1} \otimes\left(\sigma_{t}, \dot{\sigma}_{t}\right)_{\sharp} \nu_{0}$; let the potential $W_{\mu}(x, t)$ and the Lagrangian $L_{\mu, 0}(t, x, \dot{x})$ be defined at the beginning of this section. Since $\sigma_{0} \simeq \sigma_{1}$ by (5.7), the definition of $W_{\mu}$ implies that $W_{\mu}(1, x)=$ $W_{\mu}(0, x)$; thus $L_{\mu, 0}$ is 1-periodic in time.

Since $\sigma$ is minimal, it is a periodic solution of $(O D E)_{L a g}$; since the potentials $V$ and $W$ are $C^{k}$, we get that $\sigma \in C^{k+1}\left(S^{1}, L^{2}(I)\right)$; as a consequence, $W_{\mu} \in C^{k}\left(S^{1} \times S^{1}\right)$, while $V \in C^{k}\left(S^{1} \times S^{1}\right)$ by hypothesis. Using again the fact that $\sigma$ is minimal, we see as in Lemma 3.4 that $\|\dot{\sigma}\|_{C^{0}\left(\mathbf{R}, L^{2}(I)\right)}$ is bounded, independently on $\epsilon \in[0,1]$; differentiating in $(O D E)_{\text {Lag }}$, we get that the higher derivatives are bounded too. Thus, $\|\sigma\|_{C^{k}\left(\mathbf{R}, L^{2}(I)\right)}$ is bounded independently on $\epsilon \in[0,1]$; as a consequence, $\left\|W_{\mu}\right\|_{C^{k}\left(\mathbf{R} \times S^{1}\right)}$ is bounded independently on $\epsilon$. In particular, $\left\|\epsilon V+\epsilon W_{\mu}\right\|_{C^{k}\left(S^{1} \times S^{1}\right)}$ tends to zero as $\epsilon \rightarrow 0$; thus, by [15], for $\epsilon$ small and $k$ large enough, $L_{\mu, 0}$ has a KAM torus of rotation number $\omega$.

We are supposing that $\sigma$ is minimal in (5.6); by periodicity, this implies that $\sigma$ is minimal, with fixed boundary conditions, on each interval $\left[t_{0}, t_{0}+1\right]$. From Lemma 5.2 we gather that, for a.e. $x \in I, \sigma_{t} x$ is minimal for $L_{\mu, 0}$ on each interval $\left[t_{0}, t_{0}+1\right]$ for fixed boundary conditions; in particular, $: t \rightarrow \sigma_{t} x$ is an orbit of $L_{\mu, 0}$. This immediately implies that $\mu$ is invariant by the EulerLagrange flow of $L_{\mu, 0}$. Moreover, (5.8), and the fact that $\sigma_{t} \in M o n$, imply as in Sect. 3 that

$$
\lim _{t \rightarrow+\infty} \frac{\sigma_{t} x-\sigma_{0} x}{t}=\omega \quad \forall x \in I
$$

We saw above that there is $k_{0}(\tau, \gamma)$ such that, if $k \geq k_{0}(\tau, \gamma)$ and $\epsilon$ is small enough, then $L_{\mu, 0}$ has a KAM torus of frequency $\omega$. In other words, there is a $C^{1} \operatorname{map} \Phi: S^{1} \times S^{1} \rightarrow S^{1} \times S^{1} \times \mathbf{R}$ such that, denoting as usual by $\psi_{s}$ the Euler-Lagrange flow of $L_{\mu, 0}$,

$$
\psi_{s} \circ \Phi(t, x)=\Phi(t+s, x+\omega s),
$$

or $\Phi$ conjugates the rotation on $S^{1} \times S^{1}$ given by $:(t, x) \rightarrow(t+s, x+\omega s)$ to the Euler-Lagrange flow on the image of $\Phi$. We have to show that $\mu$ is the push-forward by $\Phi$ of the Lebesgue measure on $S^{1} \times S^{1}$. Since the KAM torus is conjugate to an irrational rotation, it supports just one invariant measure, i.e. the push-forward of Lebesgue. Thus, it suffices to prove that $\mu$, which we proved to be invariant, is supported on the KAM torus; equivalently, that, for each $x \in I$, the orbit $\left(t, \sigma_{t} x, \dot{\sigma}_{t} x\right)$ lies on the KAM torus. This is a consequence of (5.9) and of the fact that $: t \rightarrow \sigma_{t} x$ is an orbit. We explain why.

By [12] and [13], we know that the KAM torus is a graph; in other words, there is a Lipschitz map

$$
v: S^{1} \times S^{1} \rightarrow \mathbf{R}
$$


such that the image of $\Phi$ coincides with the graph of $v$. Moreover, the two sets

$$
A_{-}=\{(t, q, \dot{q}): \dot{q}<v(t, q)\}, \quad A_{+}=\{(t, q, \dot{q}): \dot{q}>v(t, q)\}
$$

are invariant by the flow $\phi_{s}$.

Let us call $\mathcal{T}$ the KAM torus of frequency $\omega$; it is standard that both $A_{-}$and $A_{+}$contain sequences $\mathcal{T}_{-}^{n}$ and $\mathcal{T}_{+}^{n}$ respectively of KAM tori which, as $n \rightarrow+\infty$, converge to $\mathcal{T}$. Since no orbit can cross a KAM torus, we get that, for any $z \in I$, the closure $C$ of

$$
\left\{\left(t, \sigma_{t} z, \dot{\sigma}_{t} z\right)\right\}_{t \in \mathbf{R}}
$$

either is contained in $\mathcal{T}$, or in one ot the two invariant sets $A_{ \pm}$, and at a finite distance from $\mathcal{T}$. Let us suppose by contradiction that, for some $z \in I, C$ is not contained in $\mathcal{T}$; to fix ideas, let $C \subset A_{+}$.

Let us denote by $q_{x, t}(s)$ the orbit on the KAM torus such that $q_{x, t}(t)=x$. We assert two facts:

1) if $x^{\prime}>x$, then there is a positive number $\delta\left(x^{\prime}-x\right)$, only depending on $x^{\prime}-x$, such that

$$
q_{x^{\prime}, t}(s) \geq q_{x, t}(s)+\delta\left(x^{\prime}-x\right) \quad \forall s \in \mathbf{R} .
$$

2) There is $\epsilon>0$, independent on $t \in \mathbf{R}$, such that, if $\sigma_{t} z=x$, and if $q_{x, t}(s)=q_{x, t}(t)+1=x+1$, then $\sigma_{s} z \geq q_{x, t}(s)+1+\epsilon$.

Before proving 1) and 2), we show how they imply the thesis. Let $z \in I$ and $C$ be as above; we set $\sigma_{0} z=x$; by 2), we see that, if $q_{x, 0}(s)=x+1$, then $\sigma_{s} z \geq x+1+\epsilon$. Now we set $x^{\prime}=\sigma_{s} z$; applying again point 2$)$, we have that, if $q_{x^{\prime}, s}\left(s_{1}\right)=x^{\prime}+1$, then $\sigma_{s+s_{1}} z \geq x^{\prime}+1+\epsilon$. By point 1$)$, this means that $\sigma_{s+s_{1}} z \geq q_{x, 0}\left(s+s_{1}\right)+\delta(\epsilon)+\epsilon$. Iterating, we have that

$$
\sigma_{s+s_{1}+\cdots+s_{n}} z \geq q_{x, 0}\left(s+s_{1}+\cdots+s_{n}\right)+(n-1) \delta(\epsilon)+\epsilon .
$$

This fact implies the inequality below; the equality comes from the fact that the KAM torus has rotation number $\omega$.

$$
\lim _{n \rightarrow+\infty} \frac{\sigma_{s+s_{1}+\cdots+s_{n} z}}{s+s_{1}+\cdots+s_{n}} \geq \lim _{n \rightarrow+\infty} \frac{q_{x, 0}\left(s+s_{1}+\cdots+s_{n}\right)}{s+s_{1}+\cdots+s_{n}}+\delta(\epsilon)=\omega+\delta(\epsilon) .
$$

We have reached a contradiction with (5.9).

We prove the two assertions above. To prove point 1 ), we begin to note that

$$
\Phi(t, x)=\left(t, \Phi_{x}(t, x), \Phi_{v}(t, x)\right) .
$$

Now point 1$)$ is true for the rotation $:(t, x) \rightarrow(t+s, x+\omega s)$, with $\delta\left(x^{\prime}-x\right)=$ $x^{\prime}-x$; since $\Phi$ is a conjugation, we have that $q_{x, t}(s)=\Phi_{x}(t+s, x+\omega s)$; thus, it suffices to show that the map $: x \rightarrow \Phi_{x}(t, x)$ is strictly monotone for all $t$. This follows since, by the KAM theorem, the map : $(t, x) \rightarrow\left(t, \Phi_{x}(t, x)\right)$ is close to the identity.

Since $C \subset A_{+}$is at finite distance from $\mathcal{T}$, we get from the definition of $A_{+}$that there is $a>0$ such that

$$
\dot{\sigma}_{t} z \geq v\left(\sigma_{t} z\right)+a \quad \forall x \in I, \quad \forall t \in \mathbf{R} .
$$


Let now $x \in I, t \in \mathbf{R}$ and let $q_{x, t}(s)$ be the orbit of the KAM torus with initial conditions $q_{x, t}(t)=\sigma_{t} z=x$ and $\dot{q}_{x, t}(t)=v(t, x)$. By (5.10), $\sigma_{s} z>q_{x, t}(s)$ for $s-t$ positive and small; let $(t, T)$ be the largest interval on which $\sigma_{s} z>q_{x, t}(s)$. We assert that $T=+\infty$. Indeed, if $T$ were finite, then we would have $\sigma_{T} z=q_{x, t}(T)$; together with $\sigma_{s} z>q_{x, t}(s)$ for $s \in(0, T)$, this implies that $\dot{\sigma}_{T} z \leq \dot{q}_{x, t}(T)$, contradicting (5.10). As a consequence, if $s$ is such that $q_{x, t}(s)=q_{x, t}(t)+1=\sigma_{t} z+1$, then $\sigma_{s} z \geq \sigma_{t} z+1+\epsilon$, for some $\epsilon>0$ independent on $t \in \mathbf{R}$.

\section{References}

[1] Adams, R.A.: Sobolev Spaces. Academic press, New York (1975)

[2] Ambrosio, L., Gigli, N., Savaré, G.: Gradient Flows. Birkhäuser, Basel (2005)

[3] Bernard, P., Buffoni, B.: Optimal mass transportation and Aubry-Mather theory. J. Eur. Math. Soc. 9, 85-121 (2007)

[4] Bernard, P.: Young measures, superposition and transport. Indiana Univ. Math. J. 57, 247-275 (2008)

[5] Bessi, U.: Chaotic orbits for a version of the Vlasov equation. Siam J. Math. Anal. 44, 2496-2525 (2012)

[6] Carlen, E.: Lectures on optimal mass transportation and certain of its applications, mimeographed notes (2009)

[7] Fathi, A.: Weak KAM theorem in Lagrangian dynamics, Fourth preliminary version, mimeographed notes, Lyon (2003)

[8] Gangbo, W., Tudorascu, A.: Lagrangian dynamics on an infinite-dimensional torus: a weak KAM theorem. Adv. Math. 224, 260-292 (2010)

[9] Gangbo, W., Kim, H.K., Pacini, T.: Differential forms on Wasserstein space and infinite-dimensional Hamiltonian Systems, Providence (2010)

[10] Gangbo, W., Nguyen, T., Tudorascu, A.: Hamilton-Jacobi equations in the Wasserstein space. Methods Appl. Anal. 15, 155-183 (2008)

[11] Fan, K.: Fixed point and minimax theorem in locally convex topological linear spaces. Proc. Natl. Acad. Sci. USA 38, 121-126 (1952)

[12] Mather, J.N.: Action minimizing invariant measures for positive-definite Lagrangian Systems. Math. Zeit. 207, 169-207 (1991)

[13] Mather, J.N.: Variational construction of connecting orbits. Ann. Inst. Fourier 43, 1349-1386 (1993)

[14] Nurbekian, L.: Weak KAM theory on the $d$-infinite dimensional torus, Ph.D. thesis (2012)

[15] Salamon, D., Zehnder, E.: KAM theory in configuration space. Comment. Math. Helvetici 64, 84-132 (1989) 
Ugo Bessi

Dipartimento di Matematica

Università Roma Tre

Largo S. Leonardo Murialdo

00146 Rome

Italy

e-mail: bessi@matrm3.mat.uniroma3.it

Received: 13 April 2012.

Accepted: 11 December 2012. 